\title{
Article \\ Heterosis and Combining Ability for Fruit Yield, Sweetness, $\beta$-Carotene, Ascorbic Acid, Firmness and Fusarium Wilt Resistance in Muskmelon (Cucumis melo L.) Involving Genetic Male Sterile Lines
}

\author{
Simranpreet Kaur ${ }^{1}$, Sat Pal Sharma ${ }^{1, *}$, Navraj Kaur Sarao ${ }^{2}$, Jaideep Kaur Deol ${ }^{2}$, Rupeet Gill ${ }^{1}$, \\ Kamel A. Abd-Elsalam ${ }^{3, *}$, Mousa A. Alghuthaymi ${ }^{4}$, Mohamed M. Hassan ${ }^{5}{ }^{(1)}$ and Neena Chawla ${ }^{1}$ \\ 1 Department of Vegetable Science, College of Horticulture and Forestry, Punjab Agricultural University, \\ Ludhiana 141004, India; simranpreetkaur853@gmail.com (S.K.); rupeetgill@pau.edu (R.G.); \\ chawlaneena@pau.edu (N.C.) \\ 2 School of Agricultural Biotechnology, College of Agriculture, Punjab Agricultural University, \\ Ludhiana 141004, India; navraj-soab@pau.edu (N.K.S.); jaideepkdeol@gmail.com (J.K.D.) \\ 3 Plant Pathology Research Institute, Agricultural Research Centre, Giza 12619, Egypt \\ 4 Biology Department, Science and Humanities College, Shaqra University, Alquwayiyah 11726, Saudi Arabia; \\ malghuthaymi@su.edu.sa \\ 5 Department of Biology, College of Science, Taif University, P.O. Box 11099, Taif 21944, Saudi Arabia; \\ m.khyate@tu.edu.sa \\ check for \\ * Correspondence: sharmasp@pau.edu (S.P.S.); kamel.abdelsalam@arc.sci.eg (K.A.A.-E.)
} updates

Citation: Kaur, S.; Sharma, S.P.; Sarao, N.K.; Deol, J.K.; Gill, R.; Abd-Elsalam, K.A.; Alghuthaymi, M.A.; Hassan, M.M.; Chawla, N. Heterosis and Combining Ability for Fruit Yield, Sweetness, $\beta$-Carotene, Ascorbic Acid, Firmness and Fusarium Wilt Resistance in Muskmelon (Cucumis melo L.) Involving Genetic Male Sterile Lines. Horticulturae 2022, 8, 82. https://doi.org/10.3390/

horticulturae 8010082

Academic Editor: Rosario Paolo Mauro

Received: 16 December 2021

Accepted: 11 January 2022

Published: 16 January 2022

Publisher's Note: MDPI stays neutral with regard to jurisdictional claims in published maps and institutional affiliations.

Copyright: (C) 2022 by the authors. Licensee MDPI, Basel, Switzerland. This article is an open access article distributed under the terms and conditions of the Creative Commons Attribution (CC BY) license (https:// creativecommons.org/licenses/by/ $4.0 /)$.
Abstract: Ten genetically diverse inbred lines, including two genic male sterile lines, of muskmelon (Cucumis melo L.) were crossed in a half-diallel to generate $45 \mathrm{~F}_{1}$ hybrids. These hybrids, along with the parental lines and commercial check, were evaluated for their fruit yield, level of phytochemicals and Fusarium wilt resistance. Both additive and non-additive genetic variances were important in governing the expression of all of the traits; however, the additive gene action for the fruit weight $(\mathrm{g})$, flesh thickness $(\mathrm{cm})$, rind thickness $(\mathrm{mm})$, firmness $\left(\mathrm{lb} \mathrm{inch}^{-2}\right), \beta$-carotene content $(\mathrm{mg} / 100 \mathrm{~g})$, non-additive variance for fruit yield ( $\mathrm{t} \mathrm{ha}^{-1}$ ), fruit number, total soluble solids (TSS, ${ }^{\circ} \mathrm{Brix}$ ), ascorbic acid (mg/100 g) and reaction to Fusarium wilt were comparatively more important. The parental line MM-625 was the best general combiner for fruit yield, rind thickness and $\beta$-carotene content (mg/100 g). The exotic line Riogold was the best combiner for flesh thickness and firmness. The netted inbred line MM-610 was the best general combiner for fruit weight, ascorbic acid and reaction to Fusarium wilt. The inbred lines $\mathrm{KP}_{4} \mathrm{HM}-15$ and MM-916 were the best general combiners for the number of fruits per vine and TSS. The best cross-combinations for fruit yield ha ${ }^{-1}$ and TSS were MS- $1 \times$ M-610 and Kajri $\times$ MM-904, respectively. The hybrids $\mathrm{KP}_{4} \mathrm{HM}-15 \times \mathrm{MM}$ Sel-103 and $\mathrm{KP}_{4} \mathrm{HM}-$ $15 \times$ MM-1831 recorded the highest standard heterosis for fruit yield and TSS. The landrace-derived inbred lines Kajri, MM Sel-103 and $\mathrm{KP}_{4} \mathrm{HM}-15$ produced moderate-to-highly FW-resistant hybrids. Out of the 121 SSR markers applied, 70 exhibited parental polymorphism. The markers DM0561, CMAAAGN14, TJ147, CMMS35_3, CMAGN45 and DE1337 identified specific/unique alleles in certain parental genotypes. Thus, the findings of this study revealed that the novel inbred lines can effectively be combined to generate heterotic $F_{1}$ hybrids for yield and other traits, such as rind and flesh thickness, TSS, $\beta$-carotene content and firmness. Furthermore, SSR markers can potentially be utilized to confirm the genetic diversity among the parental lines, and for the DNA fingerprinting of $\mathrm{F}_{1}$ hybrids.

Keywords: $\beta$-carotene; diallel analysis; GCA; genetic diversity; SCA; SSR markers

\section{Introduction}

Muskmelon (Cucumis melo L.) is an important cucurbitaceous crop which is adapted to warm-season regions around the world. India is among the main centers of diversity of 
melons [1]. Several genetic diversity studies have described cantalupensis, momordica and agrestris accessions of Indian origin [1-3]. Furthermore, the Indian muskmelon germplasm is a reservoir of genes for unique traits associated with fruit quality, abiotic and biotic stress tolerance [4,5], which can effectively be utilized in modern muskmelon breeding programs.

Over the past two decades, there has been a dramatic increase in the import of melon cultivars of foreign origin in India. The majority of these cultivars are not well adapted to the climate conditions of India and require very input-intensive cultivation practices. Land race-derived cultivars, such as Hara madhu, Punjab sunehri, Durgapura madhu, Pusa madhuras and Kashi madhu are still popular among Indian melon growers $[4,6]$. Several exotic melon accessions-such as Edisto, MS-1, MS-3 and Riogold-have been introduced and refined at the Punjab Agricultural University, Ludhiana [4,7]. Thus, there is a great potential for the combination of high yield potential; enhanced shelf-life traits, such as netting intensity, firmness and rind thickness; and the high phytonutrient content of exotic introductions with the abiotic and biotic stress tolerance and flavor components of landraces from India [4]. Thus, there is an urgent need to ascertain the combining ability potential and genetic parameters of the newly evolved and refined genetic resources for the development of locally adapted hybrid cultivars of muskmelon.

Heterosis refers to the phenomenon of the enhancement of crop biomass, fertility and other traits which impart superior performance to the $\mathrm{F}_{1}$ progeny over genetically diverse parents [8]. Heterosis breeding has been broadly explored and utilized in muskmelon to obtain viable intraspecific melon hybrids [9-11]. Munger [12] was the first to report hybrid vigor in muskmelon through heterosis breeding. Thus, heterosis breeding can be an effective strategy for the combination of all of the possible desirable characteristics in melon cultivars. In order to estimate the combining ability of genotypes, the diallel analysis (the half diallel design) proposed by Griffing [13] provides information about the general combining ability (GCA) of parents and the specific combining ability (SCA) of their $\mathrm{F}_{1}$ hybrids. It allows the identification of the parental lines with the higher frequency of favorable alleles through the GCA effect, and the most promising hybrid combinations through SCA effect [14]. It also provides an estimation of the gene action involved in the definition of the characteristics and presence of heterosis [15-17].

Genic male sterility (GMS) is an important mechanism utilized in muskmelon hybrid breeding [7]. A GMS inbred line MS-1 has been successfully utilized for the development of commercial $\mathrm{F}_{1}$ hybrids, Punjab hybrid [18], Punjab anmol [19] and MH-27 [20]. Furthermore, a new GMS line MM 1831, carrying the $m s-3$ gene, with thick flesh, intense netting, high $\beta$-carotene and high fruit yield was included in this study [4]. Thus, the identification of the GMS-based hybrid would reduce the expenditure of hybrid seed production by eliminating the manual emasculation of the female parent. The utilization of the GMS line will also ensure the curtailing of the impurities of the seed arising from self-pollination [7,21].

In the regions with long-term muskmelon-based cropping systems, the management of soil-borne diseases such as Fusarium wilt is becoming a major challenge [5]. Muskmelon varieties with a narrow genetic base exhibit susceptibility to various root fungal pathogens, resulting in the loss of fruit quality and yield [22]. MM Sel-103, a Fusarium wilt-resistant inbred derived from a local landrace, was utilized as male parent of two popular muskmelon hybrids, MH-27 and MH-51 [7,23]. Furthermore, Fusarium wilt-resistant genes of snapmelon and KP4 have been introgressed in the cv. Hara madhu background to develop $\mathrm{KP}_{4} \mathrm{HM}-15$ [5,22]. Thus, the information on the genetic control of Fusarium will be very useful in the development of hybrids with tolerance to the soil-borne pathogen.

Heterosis is often considered to be associated with the degree of genetic distance among parental lines [24]. Thus, the characterization of the genetic polymorphism among the parental lines at the molecular level is also critical [25]. DNA-based molecular markers provide a powerful technique for genetic diversity evaluation, the selection of diverse parental lines for hybrid development, and cultivar DNA fingerprinting [26]. Simple sequence repeat (SSR) markers offer several advantages compared with other DNA markers [27]. These markers are highly polymorphic, reproducible, multi-allelic and co- 
dominant in nature [28]. Therefore, SSR markers can be effectively used to assess the genetic divergence among breeding lines as well as $\mathrm{F}_{1}$ hybrids [29].

The overall aim of this investigation was to explore the genetic architecture of yield, its components, total soluble solids, $\beta$-carotene, ascorbic acid, firmness and Fusarium wilt resistance through diallel cross analysis. The specific objective was to choose the best heterotic parental combination for improved yield, fruit quality and Fusarium wilt resistance. The information about the genetic control of horticultural traits will definitely help melon breeders to develop $F_{1}$ hybrids with a high yield, enhanced quality and disease resistance.

\section{Materials and Methods}

\subsection{Location of the Experiment}

The experiment was carried out at the Vegetable Research Farm, Punjab Agricultural University, Ludhiana ( $30^{\circ} 54^{\prime} \mathrm{N}, 75^{\circ} 48^{\prime} \mathrm{E}$ and $248 \mathrm{~m}$ amsl), Punjab, India during 2019 and 2020. The soil of the experimental site was sandy loam. The cropping season extended from February to June.

\subsection{Genetic Materials}

Ten parental lines of muskmelon were selected based on yield, sweetness, and fruit traits such as shape, size, netting intensity, flesh thickness, rind thickness, firmness, TSS, $\beta$-carotene, ascorbic acid content and tolerance to Fusarium wilt incidence (Table 1 and Figure 1). MS-1 and MM-1831 were genic male sterile lines carrying $m s-1$ and $m s-3$ genes, respectively. The genotypes MM-1831, MM-916 and Riogold were high yielding, thick fleshed and highly netted. $\mathrm{KP}_{4} \mathrm{HM}-15$ and $\mathrm{MM}$ Sel-103 were local landraces possessing Fusarium wilt tolerance, but these genotypes lack netting and possess limited transportability. Riogold and MM- 625 had the highest levels of $\beta$-carotene and ascorbic acid content, respectively. The SSR marker-based analysis categorized the parental genotypes into three clusters, irrespective of their geographical associations (Figure 1). Kajri was a downy mildew-resistant landrace-derived inbred with good flavor and texture. These lines were chosen in order to gain a detailed understanding of their genetic potential to improve the yield and quality of muskmelon.

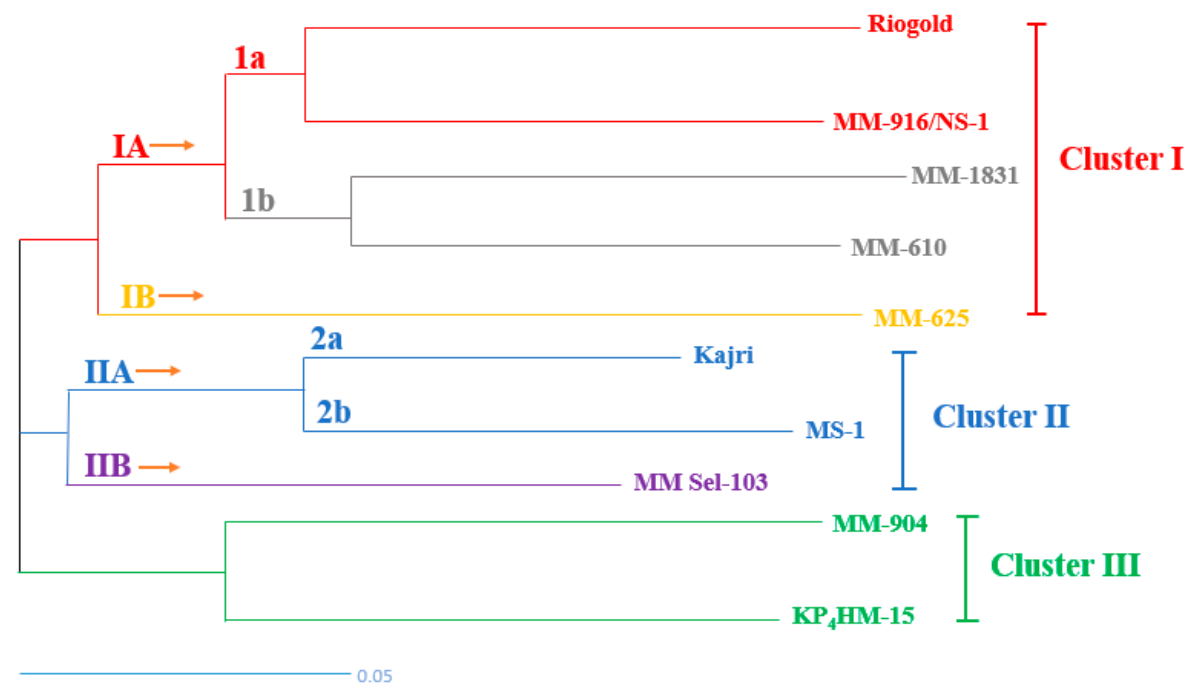

Figure 1. Dendrogram showing the neighbor joining tree of ten parental lines. 
Table 1. Particulars of the parental lines of muskmelon used in the diallel, classified according to Pitrat [30].

\begin{tabular}{|c|c|c|c|c|c|c|c|}
\hline S.No. & $\begin{array}{l}\text { Parental } \\
\text { Line }\end{array}$ & $\begin{array}{l}\text { Alternate } \\
\text { ID }\end{array}$ & Descriptive Features & Source & $\begin{array}{l}\text { Botanical } \\
\text { group/c } \\
\text { Cluster }\end{array}$ & Fruit Image & $\begin{array}{l}\text { Refer- } \\
\text { ence }\end{array}$ \\
\hline 1 & MS-1 & IC-255414 & $\begin{array}{l}\text { Oval round shape, reddish yellow } \\
\text { rind, netting with distinct sutures, } \\
\text { medium blossom scar, juicy } \\
\text { orange flesh, full slip from vine } \\
\text { upon ripening, male sterile line } \\
\text { carrying } m s-1 \text { gene }\end{array}$ & ${ }^{\mathrm{a}}$ USDA & cantalupensis/ & & [7] \\
\hline 2 & Kajri & MM 105 & $\begin{array}{l}\text { Slightly oblate shape, red striped } \\
\text { rind, sparsely netted, distinct } \\
\text { sutures, small blossom scar, juicy } \\
\text { green flesh, full slip from vine } \\
\text { upon ripening, downy mildew } \\
\text { resistant }\end{array}$ & $\begin{array}{l}\text { b PAU, } \\
\text { Ludhiana }\end{array}$ & cantalupensis/I & & [4] \\
\hline 3 & $\mathrm{KP}_{4} \mathrm{HM}-15$ & MM Var.-4 & $\begin{array}{l}\text { Oval round, creamy green rind, } \\
\text { non-netted, distinct sutures, light } \\
\text { green, medium juicy flesh, } \\
\text { Fusarium wilt resistant }\end{array}$ & $\begin{array}{c}\text { PAU, } \\
\text { Ludhiana }\end{array}$ & cantalupensis/V & & {$[5,31]$} \\
\hline 4 & MM Sel-103 & IC 623433 & $\begin{array}{l}\text { Oblate shape, yellow rind, netted, } \\
\text { distinct green sutures, orange, } \\
\text { firm flesh, Fusarium wilt tolerant }\end{array}$ & $\begin{array}{c}\text { PAU, } \\
\text { Ludhiana }\end{array}$ & cantalupensis/I & & [4] \\
\hline 5 & MM-904 & $\begin{array}{c}\text { PAU Melon } \\
904\end{array}$ & $\begin{array}{l}\text { Oval shape, light green rind, } \\
\text { non-netted distinct green sutures, } \\
\text { green, medium crisp flesh }\end{array}$ & $\begin{array}{c}\text { PAU, } \\
\text { Ludhiana }\end{array}$ & cantalupensis/ & & - \\
\hline 6 & MM-625 & $\begin{array}{l}\text { Jangpur } \\
\text { selection }\end{array}$ & $\begin{array}{l}\text { Round, reddish yellow rind, } \\
\text { intensely netted, orange flesh, } \\
\text { high firmness }\end{array}$ & $\begin{array}{c}\text { PAU, } \\
\text { Ludhiana }\end{array}$ & reticulatus/- & & - \\
\hline 7 & MM-610 & $\begin{array}{l}\text { PAU Melon } \\
610\end{array}$ & $\begin{array}{l}\text { Round, yellow green rind, } \\
\text { intensely netted, orange flesh, } \\
\text { high firmness }\end{array}$ & $\begin{array}{c}\text { PAU, } \\
\text { Ludhiana }\end{array}$ & reticulatus / & & - \\
\hline
\end{tabular}


Table 1. Cont.

\begin{tabular}{|c|c|c|c|c|c|c|c|}
\hline S.No. & $\begin{array}{l}\text { Parental } \\
\text { Line }\end{array}$ & $\begin{array}{l}\text { Alternate } \\
\text { ID }\end{array}$ & Descriptive Features & Source & $\begin{array}{l}\text { Botanical } \\
\text { group/c } \\
\text { Cluster }\end{array}$ & Fruit Image & $\begin{array}{l}\text { Refer- } \\
\text { ence }\end{array}$ \\
\hline 8 & MM-1831 & $m s-3$ & $\begin{array}{c}\text { Round, reddish yellow rind, } \\
\text { intensely netted, orange, thick } \\
\text { flesh, male sterile line carrying } \\
m s-3 \text { gene }\end{array}$ & USDA & reticulatus/II & & {$[4]$} \\
\hline 9 & MM-916 & $\begin{array}{l}\text { PAU Melon } \\
\quad 916\end{array}$ & $\begin{array}{l}\text { Oval round, yellow green rind, } \\
\text { intensely netted, orange flesh, } \\
\text { medium crisp }\end{array}$ & $\begin{array}{c}\text { PAU, } \\
\text { Ludhiana }\end{array}$ & reticulatus / & & - \\
\hline 10 & Riogold & - & $\begin{array}{l}\text { Oval round, yellow green rind, } \\
\text { intensely netted, orange flesh, } \\
\text { medium crisp, carries resistance } \\
\text { gene to race } 2 \text { of Fusarium } \\
\text { oxysporum f. sp. melonis }\end{array}$ & USDA & reticulatus/III & & [4] \\
\hline
\end{tabular}

The parental lines described at serial numbers 2-7 and 9 were developed at the Punjab Agricultural University, Ludhiana, India, and are presently maintained by Dr. Sat Pal Sharma, Principal Vegetable Breeder, Department of Vegetable Science, PAU, Ludhiana, India. ${ }^{a}$ USDA = United States Department of Agriculture. ${ }^{b}$ PAU $=$ Punjab Agricultural University, Ludhiana, India. ${ }^{c}$ The clusters refer to those described in Singh et al. [4].

\subsection{Generation of the $F_{1}$ Hybrids and Field Evaluation}

The selected 10 parents were planted in the open field in 2019, and a total of 45 crosses were generated among them in a half-diallel mating design. The $\mathrm{F}_{1}$ hybrids-including a commercial check, $\mathrm{MH}-27$-and their corresponding parents were planted in 2020 for the field evaluation. The seeds were sown in $0.025 \mathrm{~mm}$-thick polyethylene bags of size $100 \times 150 \mathrm{~mm}$ (width $\times$ depth), filled with a mixture of clay soil and farmyard manure (1:1). The seedlings were transplanted in channels at $3 \mathrm{~m}$ (row to row) and $0.60 \mathrm{~m}$ (vine to vine) spacing. The standard agronomic practices for melon crop production were followed, as described in detail in [4].

Data were recorded on the fruit weight $(\mathrm{g})$, number of fruits per vine, yield per vine $(\mathrm{kg})$, flesh thickness $(\mathrm{cm})$, rind thickness $(\mathrm{mm})$, fruit firmness, TSS $\left({ }^{\circ}\right.$ Brix $)$, and $\beta-$ carotene $(\mathrm{mg} / 100 \mathrm{~g})$. The fruit firmness $\left(\mathrm{lb} / \mathrm{inch}^{2}\right)$ was measured using a fruit penetrometer fitted with an 11-mm plunger (T.R. Turoni Srl, Via Niccolò Copernico, 26, 47122 Forlì FC, Italy). The total soluble solids were determined using a hand-held refractometer (ERMA, Bunkyo City, Tokyo, Japan), and the $\beta$-carotene content was estimated as described by Mc Collum [32].

Ascorbic acid was extracted using the method described by Heinze et al. [33]. The parental lines and $\mathrm{F}_{1}$ hybrids were evaluated against Fusarium wilt disease using the seedling root inoculation method [34]. These were scored using the $0-5$ rating scale described by Bletsos [35] $(0=$ absence of disease symptoms; $1=$ yellowing of leaves and initial wilting; 2 = a plant with continued wilting in more than one leaf; 3 = a fully wilted plant; $4=$ a plant with all of its leaves wilted and a collapsed stem; $5=$ a dead plant), and the Percent Disease Index (PDI) was calculated using the following formula:

$$
\operatorname{PDI}(\%)=\frac{\text { Sum of the all individual disease ratings }}{\text { Total number of plants observed } \times \text { Maximum grade }} \times 100
$$


Based on the PDI values, the reaction towards Fusarium wilt was determined as follows: PDI 0-10\% = Highly Resistant, 10-30\% = Resistant, 30-50\% = Moderately Resistant, $50-70 \%=$ Susceptible, and $>70 \%=$ Highly Susceptible [34].

\subsection{Molecular Characterization of Muskmelon Genotypes using Microsatellite Markers}

Genomic DNA was extracted from young expanding leaves using the CTAB (cetyl trimethyl ammonium bromide) method with some modification, as described in Doyle et al. [36]. The extracted genomic DNA was treated with polyvinyl pyrrolidone to remove the polyphenols. The DNA was quantified using a Nanodrop 1000 spectrophotometer (Thermo Scientific, Wilmington, DE, USA). The purity of the genomic DNA was estimated using the agarose gel electrophoresis methods described by Voytas et al. [37].

One hundred and twenty-one SSR markers nicely dispersed and extended over all of the twelve chromosomes of muskmelon were selected from http:/ / cucurbitgenomics.org/ pub / cucurbit/marker/ accessed on 11 December 2021, as described by Zheng et al. [38]. The DNA amplification was performed by polymerase chain reaction (PCR) in an Eppendorf thermal cycler using a final reaction volume of $20 \mu \mathrm{L}$. The PCR cycling program was comprised of an initial cycle at $94{ }^{\circ} \mathrm{C}$ for $4 \mathrm{~min}$ followed by thirty cycles of denaturation at $94{ }^{\circ} \mathrm{C}$ for $1 \mathrm{~min}$, annealing at the temperature of the primer for $1 \mathrm{~min}$, extension at $72{ }^{\circ} \mathrm{C}$ for $1 \mathrm{~min}$, and $7 \mathrm{~min}$ of final extension at $72{ }^{\circ} \mathrm{C}$. The amplified DNA fragments were separated on $6 \%$ polyacrylamide gel. The SSR bands were scored in a binary data matrix: 0 (absent) and 1 (present).

\subsection{Statistical Analysis}

The data management and analysis were performed using excel and SAS software (Version 9.3, SAS Inst., Cary, NC, USA). The means were separated using Fisher's protected LSD method. The genetic distances were calculated, and a Neighbor Joining (NJ) tree dendogram was constructed using the software DARwin (version 6.0, Darwin Technologies Ltd., London, England, UK) [39].

The analysis of variance for GCA and SCA was carried out according to Griffing's [13] method II (the parents and one set of $\mathrm{F}_{1^{\prime}} \mathrm{s}$ were included) and model 1 . The mathematical model for the combination of the ability analysis is

$$
P_{i j k}=\mu+g_{i}+g_{j}+s_{i j}+r_{k}+e_{i j k}
$$

where $\mathrm{ij}$ is the number of parents, $\mathrm{k}$ is the number of blocks or replications, $\mathrm{P}_{\mathrm{ijk}}$ is the phenotype of the $i j \mathrm{k}^{\text {th }}$ observation, $\mu$ is the population mean, $g_{i}\left(\right.$ or $\left.g_{j}\right)$ is the GCA of the $i^{\text {th }}$ or $j^{\text {th }}$ parent, $\mathrm{s}_{\mathrm{ij}}$ is the SCA of the cross between the $\mathrm{i}^{\text {th }}$ female and the $\mathrm{j}^{\text {th }}$ male parent, $\mathrm{r}_{\mathrm{k}}$ is the effect of the $\mathrm{k}^{\text {th }}$ replication, and $\mathrm{e}_{\mathrm{ijk}}$ is the environmental effect particular to the $\mathrm{ijk}^{\text {th }}$ observation.

In order to determine the GCA and SCA, the model of Diallel SAS developed by Zhang et al. [40] was used. The gene action for the inheritance of different characteristics was estimated according to the method given by Jinks and Jones [41] and Griffing [42]. Heterosis was determined as a proportion of $F_{1}$ performance in the desired direction over the better parent, by a standard check using the following formulae:

$$
\begin{gathered}
\text { Heterobeltiosis }(\mathrm{BPH}, \%)=\frac{\mathrm{F} 1-\mathrm{BP}}{\mathrm{BP}} \times 100 \\
\text { Standard heterosis }(\mathrm{SDH}, \%)=\frac{\mathrm{F} 1-\mathrm{SC}}{\mathrm{SC}} \times 100
\end{gathered}
$$

where $F_{1}$ is the mean value of the $F_{1}, B P$ is the mean performance of the better parent, and $\mathrm{SC}$ is the mean performance of the standard check for a commercial cultivar. 


\section{Results}

\subsection{Yield and Yield Components}

The analysis of variance (ANOVA) exhibited significant differences among the entries (the parents and their hybrids) for all of the traits, including yield and its component attributes (Table 2). The variances due to GCA and SCA were found to be highly significant for yield and its components, indicating the importance of both the additive and dominant effects controlling these traits. In general, the GCA/SCA ratio for the fruit yield and number of fruits per vine was less than 1.0, denoting the contribution of dominance gene action in these traits. The narrow-sense heritability was low for the yield and fruit number $(0.16$ and 0.18 , respectively), and was moderate for fruit weight $(0.44)$.

The mean fruit yield of the hybrids varied from 11.5 to $37.1 \mathrm{t} \mathrm{ha}^{-1}$, while that of the parents varied from 14.7 to $27.0 \mathrm{t} \mathrm{ha}^{-1}$ (Tables 3 and 4). Among the parental lines, the highest fruit yield was recorded in MM-1831 (27.0 $\left.\mathrm{t} \mathrm{ha}^{-1}\right)$, followed by MM-916 (26.5 $\left.\mathrm{t} \mathrm{ha}^{-1}\right)$, while the lowest fruit yield was observed in $\mathrm{KP}_{4} \mathrm{HM}-15\left(14.7 \mathrm{t} \mathrm{ha}^{-1}\right)$. The (GCA) effect for fruit yield ranged from -3.6 to 2.9 (Table 3 ). The highest positive GCA that leads to a higher fruit yield per hectare was exhibited by MM-625, and the lowest negative GCA was calculated for Kajri. For the average fruit weight, the highest positive and negative GCA effects were exhibited by MM-610 (81.1) and MM-904 (-160.8), respectively. Interestingly, the highest and lowest GCA for the number of fruits per vine was exhibited by $\mathrm{KP}_{4} \mathrm{HM}-15(0.40)$ and MM-610 (-0.33). The highest GCA for fruit yield was attributed to MM-625; however, its fruit yield was not the maximum among all of the parents (Table 3). A similar trend was observed in fruit weight and the number of fruits per vine (Table 3). The genotypes MM 916 and Kajri recorded the highest mean values for fruit weight (892.9 g) and number of fruits per vine (3.9), respectively.

The SCA effect for fruit yield ranged from -6.8 to 14.0 (Table 4 ). The highest SCA value for fruit yield was recorded for cross MS- $1 \times$ MM-610 (14.0). The magnitude of the $\mathrm{BPH}$ and SDH ranged from -49.9 to 141.5 , and from -44.1 to $79.9 \%$, respectively. The highest magnitude of heterosis for fruit yield was observed in the cross $\mathrm{KP}_{4} \mathrm{HM}-15 \times \mathrm{MM}$ Sel-103, which was 141.5\% higher than that of MM Sel-103, and 79.9\% higher than that of a commercial check, MH-27 (Figures 2 and 3). This implies that this hybrid could be utilized for selection. The highest SCA effect for fruit weight was observed in cross MS-1×MM-610. This cross recorded $1028.0 \mathrm{~g}$ average fruit weight, which was significantly higher than that of the best parent, MM-610 (733.3 g) and the commercial check MH-27 (Table 4). Thus, the highest economic heterosis for the average fruit weight was exhibited by MM- $610 \times \mathrm{MM}$ 916, which showed the second-highest SCA effect (212.4). The MM Sel-103 $\times$ MM-904 cross had the highest SCA value (1.5) for the number of fruits per vine. 
Table 2. Mean squares of the ANOVA for yield, its components, fruit quality traits and the reaction to Fusarium wilt, and the ANOVA results of the half-diallel design analysis (general and specific combining abilities) of muskmelon.

\begin{tabular}{|c|c|c|c|c|c|c|c|c|c|c|c|}
\hline $\begin{array}{l}\text { Source of } \\
\text { Variation }\end{array}$ & df & $\begin{array}{l}\text { Fruit Yield } \\
\left(\mathrm{tha}^{-1}\right)\end{array}$ & $\begin{array}{c}\text { Fruit } \\
\text { Weight(g) }\end{array}$ & $\begin{array}{c}\text { Number of } \\
\text { Fruits per } \\
\text { Vine }\end{array}$ & $\begin{array}{c}\text { Flesh Thickness } \\
(\mathrm{cm})\end{array}$ & $\begin{array}{c}\text { Rind Thickness } \\
(\mathrm{mm})\end{array}$ & $\begin{array}{l}\text { Firmness } \\
\left(\text { lb/inch }^{2}\right)\end{array}$ & $\begin{array}{l}\text { TSS } \\
\left({ }^{\circ} \text { Brix }\right)\end{array}$ & $\begin{array}{c}\beta \text {-Carotene } \\
(\mathrm{mg} / 100 \mathrm{~g})\end{array}$ & $\begin{array}{l}\text { Ascorbic Acid } \\
\text { (mg/100g) }\end{array}$ & $\begin{array}{c}\text { Reaction to } \\
\text { Fusarium } \\
\text { Wilt (PDI) }\end{array}$ \\
\hline $\operatorname{Rep}$ & 1 & 1 & 1506.4 & 0.16 & 0.07 & 0.07 & 0.06 & 1.1 & 0.01 & 1.1 & 0.1 \\
\hline Error & 54 & 0.65 & 25492.5 & 0.02 & 0.05 & 0.33 & 0.07 & 0.7 & 0.006 & 0.86 & 0.79 \\
\hline GCA & 9 & $51.7^{* *}$ & $73799.3^{* *}$ & $0.73^{* *}$ & $0.42^{* *}$ & $3.9^{* *}$ & $19.8^{* *}$ & $3.5^{* *}$ & $1.2^{* *}$ & $28.8^{* *}$ & $56.5^{* *}$ \\
\hline SCA & 45 & $42.2^{* *}$ & $15009.2^{* *}$ & $0.53^{* *}$ & $0.06^{* *}$ & $0.71^{* *}$ & $4.4^{* *}$ & $2.6^{* *}$ & $0.40^{* *}$ & $20.7^{\text {** }}$ & $50.2^{* *}$ \\
\hline Error & 54 & 0.32 & 907 & 0.01 & 0.02 & 0.16 & 0.03 & 0.35 & 0.003 & 0.43 & 0.39 \\
\hline$\sigma_{s}^{2}$ & - & 41.9 & 14102.2 & 0.52 & 0.03 & 0.55 & 4.4 & 2.3 & 0.40 & 20.2 & 49.8 \\
\hline$\sigma^{2} \mathrm{E}$ & - & 0.32 & 907.07 & 0.01 & 0.03 & 0.16 & 0.03 & 0.35 & 0.003 & 0.43 & 0.39 \\
\hline$\sigma^{2} \mathrm{~A}$ & - & 8.5 & 12148.7 & 0.12 & 0.06 & 0.63 & 3.2 & 0.53 & 0.20 & 4.7 & 9.3 \\
\hline$\sigma^{2}{ }_{D}^{A}$ & - & 41.9 & 14102.2 & 0.52 & 0.03 & 0.55 & 4.4 & 2.3 & 0.40 & 20.2 & 49.8 \\
\hline$\sigma^{2} \mathrm{P}$ & - & 50.8 & 27157.9 & 0.65 & 0.13 & 1.3 & 7.7 & 3.2 & 0.61 & 25.4 & 59.5 \\
\hline$\sigma^{2} g / \sigma^{2}{ }_{s}$ & - & 0.102 & 0.43 & 0.11 & 0.89 & 0.58 & 0.37 & 0.11 & 0.25 & 0.12 & 0.09 \\
\hline$h^{2} \mathrm{n}$ & - & 0.16 & 0.44 & 0.18 & 0.49 & 0.47 & 0.42 & 0.17 & 0.33 & 0.18 & 0.15 \\
\hline$h^{2}{ }_{b}$ & - & 0.99 & 0.96 & 0.98 & 0.77 & 0.87 & 0.99 & 0.89 & 0.99 & 0.98 & 0.99 \\
\hline
\end{tabular}


Table 3. Least square means and estimates of the general combining ability (GCA) effects in a half-diallel analysis of 10 muskmelon parental lines.

\begin{tabular}{|c|c|c|c|c|c|c|c|c|c|c|c|c|c|c|c|c|c|c|c|c|}
\hline \multirow[t]{2}{*}{ Parents } & \multicolumn{2}{|c|}{$\begin{array}{l}\text { Fruit Yield } \\
\left(\mathrm{t} \mathrm{ha} \mathbf{a}^{-1}\right)\end{array}$} & \multicolumn{2}{|c|}{ Fruit Weight (g) } & \multicolumn{2}{|c|}{$\begin{array}{l}\text { Number of } \\
\text { Fruits per Vine }\end{array}$} & \multicolumn{2}{|c|}{$\begin{array}{l}\text { Flesh } \\
\text { Thickness (cm) }\end{array}$} & \multicolumn{2}{|c|}{$\begin{array}{l}\text { Rind Thickness } \\
\text { (mm) }\end{array}$} & \multicolumn{2}{|c|}{$\begin{array}{l}\text { Firmness } \\
\left(\mathrm{lb} / \text { inch }^{2}\right)\end{array}$} & \multicolumn{2}{|c|}{ TSS $\left({ }^{\circ}\right.$ Brix) } & \multicolumn{2}{|c|}{$\begin{array}{l}\beta \text {-Carotene } \\
(\mathrm{mg} / 100 \mathrm{~g})\end{array}$} & \multicolumn{2}{|c|}{$\begin{array}{l}\text { Ascorbic Acid } \\
\text { (mg/100 g) }\end{array}$} & \multicolumn{2}{|c|}{$\begin{array}{c}\text { Reaction to } \\
\text { Fusarium Wilt } \\
\text { (PDI) }\end{array}$} \\
\hline & Mean & GCA & Mean & GCA & Mean & GCA & Mean & GCA & Mean & GCA & Mean & GCA & Mean & GCA & Mean & GCA & Mean & GCA & Mean & GCA \\
\hline MS-1 & 21.6 & $-0.73^{* *}$ & 687.5 & $-33.3 *$ & 3.3 & -0.02 & 2.1 & -0.05 & 4.5 & $0.40 * *$ & 14.5 & $1.3 * *$ & 8.7 & $-0.33 *$ & 1.6 & -0.01 & 26.2 & $1.5^{* *}$ & 36.0 & -0.14 \\
\hline Kajri & 20.5 & $-3.6^{* *}$ & 550.0 & $-83.7^{* *}$ & 3.9 & $-0.18^{* *}$ & 2.2 & $-0.13^{* *}$ & 2.9 & -0.54 ** & 11.0 & $0.66^{* *}$ & 8.8 & $-0.77^{* *}$ & 0.55 & $-0.26^{* *}$ & 21.1 & & 38.2 & $-2.3^{* *}$ \\
\hline $\mathrm{KP}_{4} \mathrm{HM}-15$ & 14.7 & $2.3^{* *}$ & 636.7 & $-35.1^{* *}$ & 2.5 & $0.40^{* *}$ & 2.3 & $-0.23^{* *}$ & 3.3 & $-0.83^{* *}$ & 5.8 & $-2.1^{* *}$ & 11.2 & $0.34 *$ & 0.63 & $-0.42 * *$ & 17.8 & $-2.1^{* *}$ & 23.5 & $1.3^{* *}$ \\
\hline $\begin{array}{c}\text { MM } \\
\text { Sel-103 }\end{array}$ & 15.3 & $1.8^{* *}$ & 691.1 & $24.4^{* *}$ & 2.3 & $0.15^{* *}$ & 2.2 & 0.06 & 6.3 & 0.12 & 7.9 & $-1.08^{* *}$ & 9.1 & -0.05 & 1.7 & $0.06^{* *}$ & 11.7 & $-2.7^{* *}$ & 25.9 & $-2.8 * *$ \\
\hline MM-904 & 14.9 & $-2.5^{* *}$ & 635.0 & $-160.8^{* *}$ & 2.5 & $0.35^{* *}$ & 1.9 & -0.26 ** & 3.0 & $-0.86^{* *}$ & 5.4 & $-1.7^{* *}$ & 9.0 & $-0.50^{* *}$ & 0.52 & $-0.53 * *$ & 25.3 & $0.73^{* *}$ & 37.5 & $2.0 * *$ \\
\hline MM-625 & 18.5 & $2.9^{* *}$ & 849.7 & $74.2^{* *}$ & 2.3 & $0.10^{* *}$ & 2.6 & $0.17^{* *}$ & 7.0 & $0.90^{* *}$ & 10.6 & $0.79^{* *}$ & 10. & 0.14 & 1.6 & $0.48^{* *}$ & 27 & $1.0^{* *}$ & 33.6 & $1.1^{* *}$ \\
\hline MM-610 & 15.6 & 0.04 & 733.3 & 81.1 & 2.3 & $-0.33^{* *}$ & 2.2 & 0.04 & 4.5 & 0.40 & 8.9 & -0.3 & 10. & -0.14 & 2.5 & 0.2 & 26 & 1.8 & 19.5 & $-3.5^{* *}$ \\
\hline MM-916 & 26.5 & $-0.47^{* * *}$ & 892.9 & $25.9^{* *}$ & 3.1 & $-0.16^{* *}$ & 2.3 & $-0.09 *$ & 4.3 & -0.05 & 5.8 & 0.65 ** & 9.0 & $1.0^{* *}$ & 1.4 & $0.18^{* *}$ & 21 & $0.40^{*}$ & 37.6 & $2.4^{* *}$ \\
\hline Riogold & 23.2 & $0.50 * *$ & 839.3 & $75.7^{* *}$ & 2.9 & $-0.21^{* *}$ & 3.1 & $0.30 * *$ & 5.3 & 0.17 & 10.7 & $1.5^{* *}$ & 9.1 & -0.3 & 2.3 & $0.24^{* *}$ & 19.0 & $-1.5^{* *}$ & 34.5 & $1.7^{* *}$ \\
\hline$(p<0.05)$ & 1.6 & 0.35 & 83.4 & 18.6 & 0.31 & 0.06 & 0.48 & 0.10 & 1.1 & 0.25 & 0.59 & 0.11 & 1.6 & 0.36 & 0.16 & 0.03 & 1.8 & 0.40 & 1.8 & 0.39 \\
\hline
\end{tabular}

* Significant at the $5 \%$ level; ** significant at the $1 \%$ level. Abbreviations: SCA—specific combining ability; BPH—better parent heterosis; SDH—standard heterosis. 
Table 4. Estimation of the mean, specific combining ability, better parent and standard heterosis (\%) over the commercial check MH-27 for the fruit yield, number of fruits per vine and fruit weight of muskmelon.

\begin{tabular}{|c|c|c|c|c|c|c|c|c|c|c|c|c|}
\hline \multirow{2}{*}{ Hybrid } & \multicolumn{4}{|c|}{ Fruit Yield (t ha $\left.{ }^{-1}\right)$} & \multicolumn{4}{|c|}{ Number of Fruits per Vine } & \multicolumn{4}{|c|}{ Fruit Weight (g) } \\
\hline & Mean & SCA & ВРH & SDH & Mean & SCA & ВРН & SDH & Mean & SCA & ВРH & SDH \\
\hline $1 \times 2$ & 21.4 & $4.3^{* *}$ & -1.1 & 3.8 & 3.0 & 0.15 & $-24.7^{* *}$ & $-12.5^{* *}$ & 758.4 & $122.0 * *$ & 10.3 & $18.7^{* *}$ \\
\hline $1 \times 3$ & 20.8 & $-2.2 * *$ & -3.7 & 1.1 & 3.0 & $-0.41^{* *}$ & $-9.9 *$ & $-12.1^{* *}$ & 734.6 & 49.6 & 6.8 & $14.9 *$ \\
\hline $1 \times 4$ & 17.8 & $-4.7 * *$ & $-17.5^{* *}$ & $-13.3^{* *}$ & 3.0 & -0.16 & $-9.9 *$ & $-12.1^{* *}$ & 629.7 & $-14.9^{* *}$ & -8.9 & -1.4 \\
\hline $1 \times 5$ & 17.0 & $-1.1 *$ & $-21.5 * *$ & $-17.5^{* *}$ & 3.0 & $-0.41^{* *}$ & $-11.4 *$ & $-13.6^{* *}$ & 609.2 & 49.9 & -11.3 & -4.6 \\
\hline $1 \times 6$ & 25.9 & $2.2^{* *}$ & $19.7^{* *}$ & $25.7^{* *}$ & 3.3 & 0.13 & -2.4 & -4.8 & 843.8 & 49.4 & -0.69 & $32.2 * *$ \\
\hline $1 \times 7$ & 35.1 & $14^{* *}$ & $62.5^{* *}$ & $70.6^{* *}$ & 3.6 & $0.94^{* *}$ & 8.7 & 6.0 & 1028 & $226.0 * *$ & $40.2^{* *}$ & $60.9^{* *}$ \\
\hline $1 \times 8$ & 13.7 & $-6.7^{* *}$ & $-49.2 * *$ & $-33.3^{* *}$ & 2.7 & $-0.22 *$ & $-19.3^{* *}$ & $-21.3^{* *}$ & 541.3 & $-210.0 * *$ & $-37.5^{* *}$ & -15.2 * \\
\hline $1 \times 10$ & 15.0 & $-6.2 * *$ & $-35.6^{* *}$ & $-27.3^{* *}$ & 2.3 & $-0.53^{* *}$ & $-31.9 * *$ & $-33.6^{* *}$ & 699.5 & $-96.3^{* *}$ & $-16.6^{* *}$ & 9.4 \\
\hline $2 \times 3$ & 13.9 & $-6.1^{* *}$ & $-32.2^{* *}$ & $-32.4^{* *}$ & 2.6 & $-0.65^{* *}$ & $-34.4^{* *}$ & $-23.8^{* *}$ & 567.1 & $-67.4^{*}$ & -10.9 & -11.2 \\
\hline $2 \times 4$ & 17.0 & $-2.6^{* *}$ & $-17.4^{* *}$ & $-17.6^{* *}$ & 2.2 & $-0.85^{* *}$ & $-45.7^{* *}$ & $-37.0 * *$ & 837.5 & $143.0 * *$ & $21.2 * *$ & $31.1^{* *}$ \\
\hline $2 \times 5$ & 11.5 & $-3.6^{* *}$ & $-43.9^{* *}$ & $-44.1^{* *}$ & 2.5 & $-0.70^{* *}$ & $-36.9^{* *}$ & $-26.7^{* *}$ & 488.9 & -19.9 & $-23.0^{* *}$ & $-23.4^{* *}$ \\
\hline $2 \times 6$ & 22.2 & $1.5^{* *}$ & $8.1^{*}$ & 7.7 & 3.6 & $0.60^{* *}$ & $-10.2 *$ & 4.2 & 659.8 & $-84.2^{* *}$ & $-22.3^{* *}$ & 3.2 \\
\hline $2 \times 7$ & 13.4 & $-4.4^{* *}$ & $-34.9^{* *}$ & $-35.1^{* *}$ & 2.3 & $-0.21 *$ & $-41.9^{* *}$ & $-32.6^{* *}$ & 615.4 & $-135.0 * *$ & $-16.1^{* *}$ & -3.6 \\
\hline $2 \times 8$ & 13.5 & $-4.0^{* *}$ & $-49.9 * *$ & $-34.3^{* *}$ & 2.2 & $-0.58^{* *}$ & $-45.4^{* *}$ & $-36.6^{* *}$ & 670.6 & -30.6 & $-22.6^{* *}$ & 4.9 \\
\hline $2 \times 9$ & 14.0 & $-3.3^{* *}$ & $-47.4^{* *}$ & $-32.2^{* *}$ & 2.3 & $-0.43^{* *}$ & $-43.2^{* *}$ & $-34.1^{* *}$ & 661.2 & -34.5 & $-25.9^{* *}$ & 3.4 \\
\hline $2 \times 10$ & 23.8 & $5.5^{* *}$ & 2.4 & $15.7^{* *}$ & 2.7 & 0.099 & $-31.0 * *$ & $-19.9 * *$ & 924.3 & $178.0 * *$ & 10.1 & $44.6^{* *}$ \\
\hline $3 \times 5$ & 32.6 & $11.4^{* *}$ & $117.9^{* *}$ & $58.3^{* *}$ & 5.0 & $1.16^{* *}$ & $98.0^{* *}$ & $44.9 * *$ & 697.5 & $140.0^{* *}$ & 9.5 & 9.1 \\
\hline $3 \times 6$ & 36.0 & $9.3^{* *}$ & $93.8^{* *}$ & $74.9^{* *}$ & 4.2 & $0.62^{* * *}$ & $70.0^{* *}$ & $21.9^{* *}$ & 916.8 & $124.2 * *$ & 7.9 & $43.5^{* *}$ \\
\hline $3 \times 7$ & 16.9 & $-6.8^{* *}$ & 7.9 & $-17.8^{* *}$ & 2.5 & $-0.56^{* *}$ & 3.4 & $-25.7^{* *}$ & 707.1 & $-92.3^{* *}$ & -3.5 & 10.6 \\
\hline $3 \times 8$ & 27.4 & $3.8^{* *}$ & 1.3 & $32.8^{* *}$ & 3.8 & $0.50^{* *}$ & $16.2 * *$ & $12.3^{* *}$ & 756.2 & 6.3 & $-12.7 *$ & $18.3^{* *}$ \\
\hline $3 \times 9$ & 18.1 & $-5.2 * *$ & $-32.1^{* *}$ & $-12.3^{* *}$ & 3.2 & -0.09 & 0.79 & -7.0 & 603.5 & $-140.7^{* *}$ & $-32.4^{* *}$ & -5.5 \\
\hline $3 \times 10$ & 31.6 & $7.3^{* *}$ & $35.9^{* *}$ & $53.6^{* *}$ & 4.5 & $1.2 * *$ & $52.1^{* *}$ & $30.7 * *$ & 750.4 & -43.6 & $-10.6^{*}$ & $17.4^{*}$ \\
\hline $4 \times 5$ & 27.31 & $6.5^{* *}$ & $78.0^{* *}$ & $32.5^{* *}$ & 5.1 & $1.5^{* *}$ & $104.6^{* *}$ & $49.7^{* *}$ & 566.5 & -50.5 & $-18.0^{* *}$ & -11.3 \\
\hline $4 \times 6$ & 34.6 & $8.3^{* *}$ & $86.1^{* *}$ & $67.8^{* *}$ & 4.2 & $0.85^{* *}$ & $76.6^{* *}$ & $21.5^{* *}$ & 883.9 & 31.6 & 4.0 & $38.3^{* *}$ \\
\hline $4 \times 7$ & 33.3 & $9.9^{* *}$ & $112.2^{* *}$ & $61.5^{* *}$ & 3.5 & $0.68^{* *}$ & $50.2^{* *}$ & 3.3 & 997.9 & $138.7^{* *}$ & $36.1^{* *}$ & $56.1 * *$ \\
\hline $4 \times 8$ & 22.0 & $-1.0 *$ & $-18.4^{* *}$ & 6.9 & 2.3 & $-0.75^{* *}$ & $-29.3^{* *}$ & $-31.7^{* *}$ & 1000.6 & $191.1^{* *}$ & $15.4^{* *}$ & $56.6^{* *}$ \\
\hline $4 \times 9$ & 15.7 & $-7.1^{* *}$ & $-40.9^{* *}$ & $-23.8^{* *}$ & 2.7 & $-0.35^{* *}$ & $-15.2^{* *}$ & $-21.8^{* *}$ & 622.2 & $-181.0^{* *}$ & $-30.3^{* *}$ & -2.6 \\
\hline $4 \times 10$ & 22.8 & -0.9 & -1.8 & $10.8^{* *}$ & 3.0 & 0.04 & 2.7 & $-11.7^{*}$ & 801.9 & -51.7 & -4.4 & $25.5^{* *}$ \\
\hline $5 \times 6$ & 17.7 & $-4.0 * *$ & -4.7 & $-14.1^{* *}$ & 3.5 & 0.01 & $40.0 * *$ & 2.4 & 543.9 & $-122.0 * *$ & $-35.9 * *$ & $-14.8^{*}$ \\
\hline $5 \times 7$ & 16.7 & $-2.2 * *$ & 6.2 & $-19.1^{* *}$ & 3.0 & -0.09 & $18.0^{* *}$ & $-13.6^{* *}$ & 597.6 & $-76.1^{* *}$ & $-18.5^{* *}$ & -6.4 \\
\hline
\end{tabular}


Table 4. Cont.

\begin{tabular}{|c|c|c|c|c|c|c|c|c|c|c|c|c|}
\hline \multirow{2}{*}{ Hybrid } & \multicolumn{4}{|c|}{ Fruit Yield (t ha-1) } & \multicolumn{4}{|c|}{ Number of Fruits per Vine } & \multicolumn{4}{|c|}{ Fruit Weight (g) } \\
\hline & Mean & SCA & ВРH & SDH & Mean & SCA & ВРН & SDH & Mean & SCA & ВРН & SDH \\
\hline $5 \times 8$ & 21.9 & $3.2^{* *}$ & $-18.9^{* *}$ & 6.3 & 4.6 & $1.3^{* *}$ & $38.9^{* *}$ & $34.2 * *$ & 505.6 & $-118.0 * *$ & $-41.6^{* *}$ & $-20.8^{* *}$ \\
\hline $5 \times 9$ & 14.1 & $-4.2^{* *}$ & $-46.7^{* *}$ & $-31.3^{* *}$ & 3.3 & 0.11 & 5.7 & -2.4 & 449.4 & $-169.0 * *$ & $-49.6^{* *}$ & $-29.6^{* *}$ \\
\hline $5 \times 10$ & 15.9 & $-3.4^{* *}$ & $-31.8^{* *}$ & $-22.9 * *$ & 2.7 & $-0.49^{* *}$ & -9.0 & $-21.8^{* *}$ & 628.9 & -39.4 & $-25.1^{* *}$ & -1.5 \\
\hline $6 \times 7$ & 28.4 & $4.0 * *$ & $52.8^{* *}$ & $37.8^{* *}$ & 3.0 & 0.15 & $27.4^{* *}$ & $-13.6^{* *}$ & 1018.9 & $109.9 * *$ & $19.9 * *$ & $59.4^{* *}$ \\
\hline $6 \times 8$ & 23.5 & -0.6 & $-12.9^{* *}$ & $14.1^{* * *}$ & 2.9 & -0.13 & -12.1 * & -15.0 ** & 859.8 & 0.5 & -0.8 & $34.5^{* *}$ \\
\hline $6 \times 9$ & 25.3 & $1.4^{* *}$ & -4.5 & $23.1^{* *}$ & 3.3 & $0.28^{* *}$ & 3.6 & -4.3 & 822.2 & -31.5 & -7.93 & $28.6^{* *}$ \\
\hline $6 \times 10$ & 19.9 & $-4.9^{* *}$ & $-14.4^{* *}$ & -3.2 & 2.3 & $-0.65^{* *}$ & $-22.8^{* *}$ & $-33.6^{* *}$ & 931 & 27.5 & 9.5 & $45.7^{* *}$ \\
\hline $7 \times 9$ & 23.6 & $2.6^{*}$ & $-11.1^{* *}$ & $14.6^{* *}$ & 2.3 & -0.2 & $-26.0 * *$ & $-31.7^{* *}$ & 1073.1 & $212.4 * *$ & $20.2^{* *}$ & $67.9^{* *}$ \\
\hline $7 \times 10$ & 19.0 & $-2.9^{* *}$ & $-18.3^{* *}$ & -7.7 & 2.3 & $-0.21^{*}$ & $-22.8^{* *}$ & $-33.6^{* *}$ & 889.2 & -21.1 & 5.9 & $39.2 * *$ \\
\hline $8 \times 9$ & 20.3 & -0.4 & $-24.9^{* *}$ & -1.6 & 2.5 & $-0.31^{* *}$ & $-25.7^{* *}$ & -28.2 ** & 875.9 & $64.9 *$ & -1.9 & $37.1^{* *}$ \\
\hline $8 \times 10$ & 18.3 & $-3.4^{* *}$ & $-32.2^{* *}$ & $-11.1^{* *}$ & 2.3 & $-0.4^{* * *}$ & $-31.3^{* *}$ & $-33.6^{* *}$ & 855 & -5.7 & -1.3 & $33.8^{* *}$ \\
\hline $9 \times 10$ & 28.9 & $7.4^{* *}$ & $8.8^{* *}$ & $40.4^{* *}$ & 3.0 & $0.29^{* *}$ & -6.3 & $-13.6^{* *}$ & 1037.9 & $182.0 * *$ & $16.2^{* *}$ & $62.4^{* *}$ \\
\hline $\begin{array}{c}\mathrm{LSD}(p \leq \\
0.05)\end{array}$ & 1.6 & 1.0 & 1.6 & 1.6 & 0.31 & 0.20 & 0.31 & 0.31 & 83.4 & 55.9 & 85.84 & 85.8 \\
\hline
\end{tabular}

${ }^{*}$ Significant at the $5 \%$ level; ${ }^{* *}$ significant at the $1 \%$ level. Abbreviations: SCA—specific combining ability; BPH—better parent heterosis; SDH—standard heterosis. 


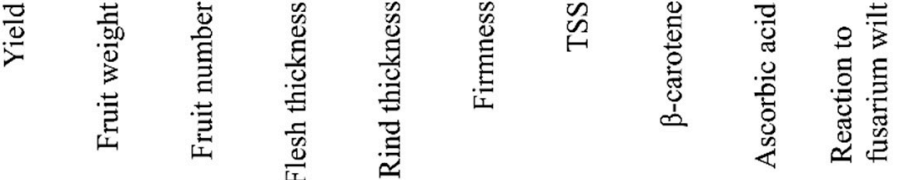

KP4HM-15×MM Scl- 1 KP4HM-15×MM- 625

MM Sel-103×MM-625

MM Sel-103×MM-610

KP4HM-15×MM-904

KP4HM-15×Riogold

MM $916 \times$ Riogold

MM- $625 \times$ MM- 610

KP4HM-15×MM-1831

MM Scl-103×MM-904

MS- $1 \times$ MM- 625

MM-625×MM 916

Kajri $\times$ Riogold

MM-610×MM 916

MM- $625 \times$ MM- 1831

MM Sel-103×Riogold

Kajri $\times$ MM-625

MM Scl-103×MM-183

MM-904×MM-1831

MS-1 $\times$ Kajri

MS- $1 \times \mathrm{KP} 4 \mathrm{HM}-15$

MM- $1831 \times$ MM 916

MM-625×Riogold

MM-610×Riogold

MM-610 $\times$ MM- 1831

MM-1831×Riogold

KP4HM-15×MM 916

MS- $1 \times$ MM Sel-103

MM-904×MM- 625

MS- $1 \times$ MM-904

MS-1 $\times$ MM 916

Kajri $\times$ MM Sel-103

KP4HM-15×MM-610

MM-904×MM-610

MM-904×Riogold

MM Sel-103×MM 916

MS-1×Riogold

MM-904×MM 916

Kajri $\times$ MM 916

Kajri $\times$ KP4HM- 15

MS- $1 \times$ MM- 183

Kajri $\times$ MM-1831

Kajri $\times$ MM-610

Kajri×MM-904
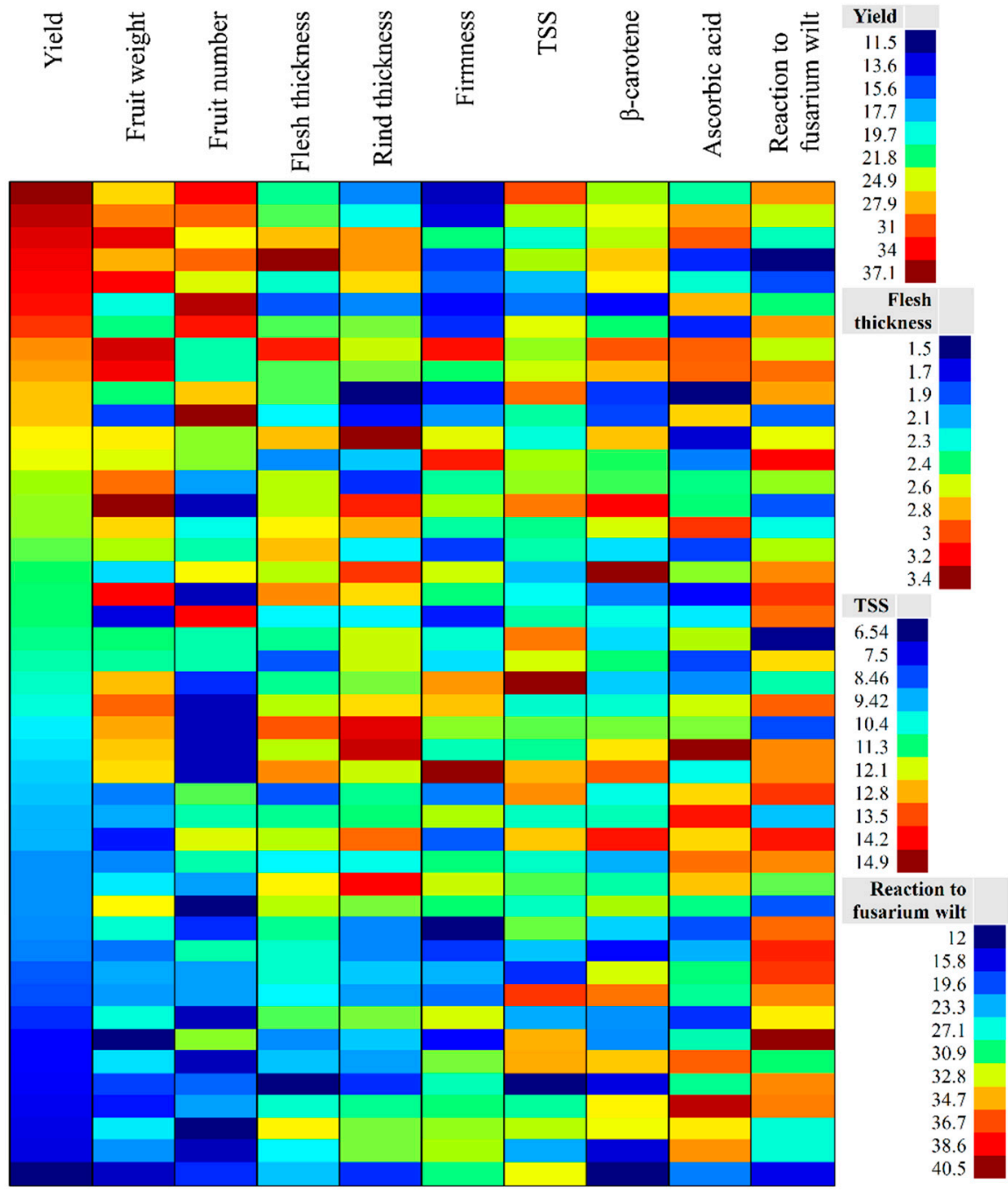

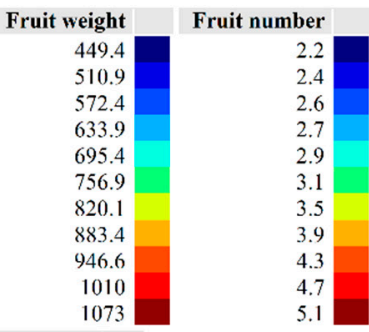

\begin{tabular}{r|r}
$\begin{array}{r}\text { Rind } \\
\text { thickness }\end{array}$ & Firmness \\
\hline
\end{tabular}

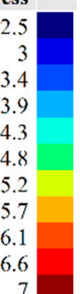

$\beta$-carotene

\begin{tabular}{r|r|}
\hline 0.2 & \\
0.5 & \\
0.8 & \\
1.1 \\
1.4 \\
1.7 \\
2 \\
2.4 \\
2.7 \\
3.1 \\
3.4 & \\
\hline
\end{tabular}

Ascorbic

\begin{tabular}{l}
9.33 \\
11.1 \\
12.9 \\
14.7 \\
16.4 \\
18.2 \\
19.9 \\
21.7 \\
23.4 \\
25.1 \\
26.9 \\
\hline
\end{tabular}

Figure 2. Cell plot exhibiting the variability among 45 hybrids of muskmelon for yield, fruit traits and reaction to Fusarium wilt. 


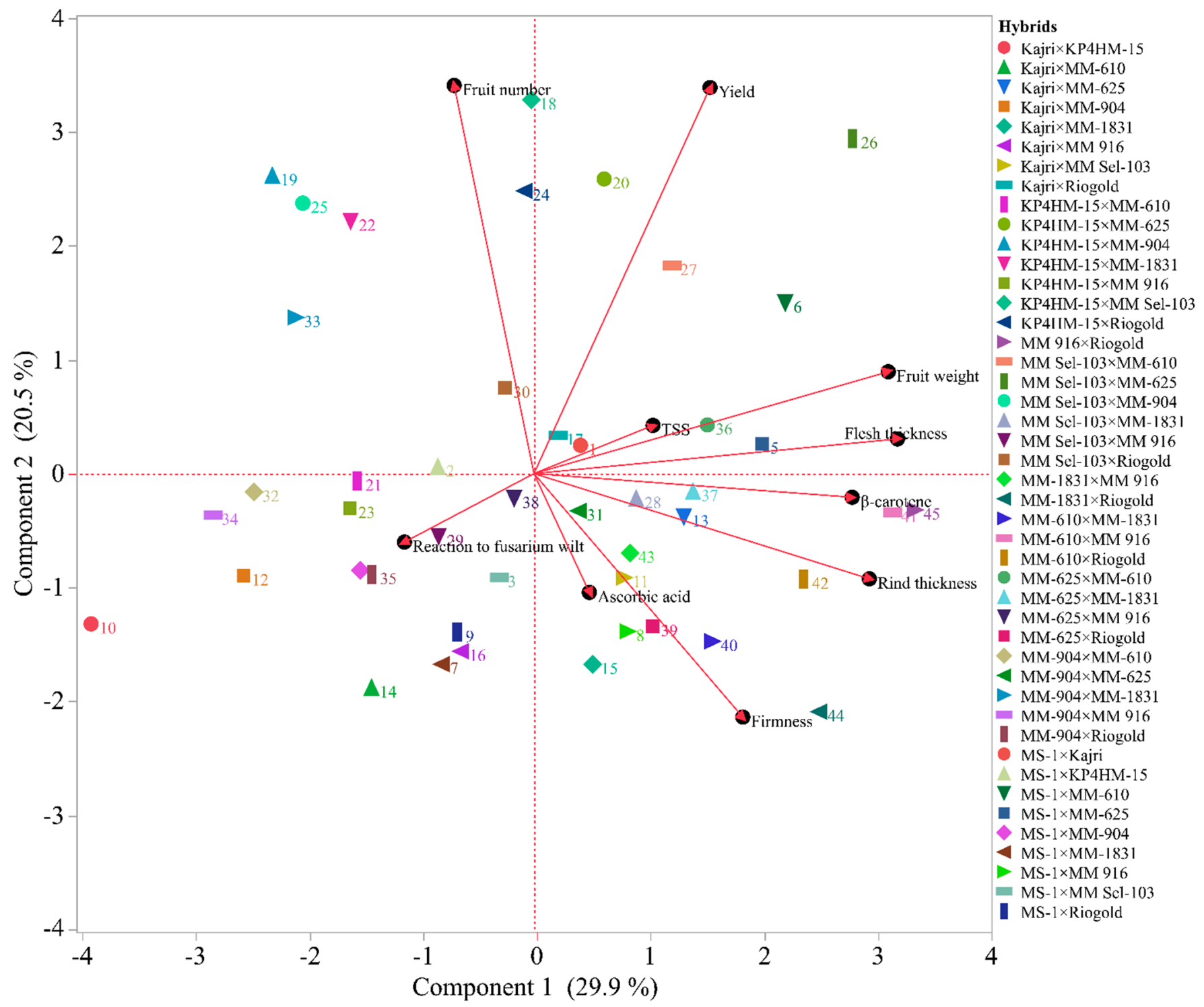

Figure 3. Genotype-by-trait biplot of 45 muskmelon hybrids based upon yield, fruit traits and reaction to Fusarium wilt.

\subsection{Fruit Quaility Traits}

The genotypes exhibited significant differences for all of the fruit quality-related traits (Table 2). Highly significant GCA and SCA effects were observed for all of the fruit characteristics which revealed that both additive and non-additive (dominance) effects control the action of these traits.

The ANOVA exhibited significant differences among the genotypes (parents and their hybrids) for all of the fruit quality traits (Table 2). The variances due to GCA and SCA were found to be highly significant for flesh thickness, rind thickness, firmness, TSS, $\beta$-carotene and ascorbic acid, indicating the importance of both the additive and dominant effects controlling these traits. The GCA/SCA ratio for flesh thickness was close to 1.0, implying the contribution of both additive and dominance gene action on this trait. Meanwhile, the GCA/SCA ratios for rind thickness, firmness, TSS, $\beta$-carotene and ascorbic acid were less than 1.0, denoting the contribution of dominance gene action in these traits. The narrow-sense heritability was low for TSS and ascorbic acid ( 0.17 and 0.18 , respectively), while it was moderate for flesh thickness, rind thickness, firmness and $\beta$-carotene $(0.49$, $0.47,0.42$ and 0.33 , respectively). 
The highest GCA value for flesh thickness was shown by Riogold (0.30), and the highest negative GCA was calculated for MM-904 (-0.26) (Table 3). Among the parental lines, Riogold exhibited the highest flesh thickness $(3.1 \mathrm{~cm})$, while the thinnest flesh was observed in MM-904 $(1.9 \mathrm{~cm})$. The highest SCA value for flesh thickness was observed in the cross MM Sel-103 $\times$ MM-625 (Table 5, Figures 2 and 3). This cross exhibited the highest heterosis over the better parent, MM-625 (28.6\%), and also exhibited SDH over the commercial check, MH-27 (74.3\%). In addition, the hybrids' combinations, MM- $610 \times$ Riogold, MM-183 $\times$ Riogold, and MM-916 $\times$ Riogold were also found to be promising for this trait, exhibiting $54.4,51.0$ and $60.6 \% \mathrm{SDH}$, respectively.

Table 5. Estimation of the mean, specific combining ability, better parent and standard heterosis (\%) over commercial check MH-27 for the flesh thickness, rind thickness and firmness of muskmelon.

\begin{tabular}{|c|c|c|c|c|c|c|c|c|c|c|c|c|}
\hline \multirow{2}{*}{ Hybrid } & \multicolumn{4}{|c|}{ Flesh Thickness (cm) } & \multicolumn{4}{|c|}{ Rind Thickness (cm) } & \multicolumn{4}{|c|}{ Firmness $\left(\mathrm{lb} /\right.$ inch $\left.^{2}\right)$} \\
\hline & Mean & SCA & BPH & SDH & Mean & SCA & BPH & $\mathrm{SDH}$ & Mean & SCA & BPH & SDH \\
\hline $1 \times 2$ & 2.4 & 0.13 & 6.7 & 23.5 & 5.2 & 0.51 & 14.7 & 10.7 & 7.1 & $-2.7 * *$ & $-50.6^{* *}$ & $-52.3^{* *}$ \\
\hline $1 \times 3$ & 1.9 & -0.21 & -14 & 0.26 & 5.2 & $0.80 *$ & 14.7 & 10.7 & 6.6 & $-0.48^{* *}$ & $-54.1^{* *}$ & $-35.7^{* *}$ \\
\hline $1 \times 4$ & 2.4 & -0.05 & 7.6 & 24.3 & 4.8 & -0.48 & $-23.7 *$ & 3.5 & 9. & $0.82 * *$ & $-37.9 * *$ & $-45.2^{* *}$ \\
\hline $1 \times 5$ & 2.2 & 0.05 & 3.8 & 12.9 & 4.3 & 0.007 & -3.6 & -7.0 & 7.7 & 0.2 & $-46.9 * *$ & -28.5 * \\
\hline $1 \times 6$ & 2.8 & 0.24 & 7.1 & $45.0 * *$ & 7.0 & $0.90 *$ & 0.0 & 50.0 ** & 9.5 & $-0.50 * *$ & $-34.1^{* *}$ & -19.0 \\
\hline $1 \times 7$ & 2.8 & $0.41 *$ & $24.3 *$ & $46.9^{* *}$ & 5.8 & 0.24 & 29.6 * & $25.1 *$ & 7.7 & $-1.2^{* *}$ & $-46.9^{* *}$ & -16.6 \\
\hline $1 \times 8$ & 2.3 & -0.23 & $-18.5^{*}$ & 20.9 & 4.7 & -0.81 * & -19.9 * & 0.0 & 7.7 & $-1.8^{* *}$ & $-46.5^{* *}$ & $-30.9^{*}$ \\
\hline $1 \times 9$ & 2.7 & $0.37^{*}$ & 13.4 & $38.1^{* *}$ & 6.5 & $1.3^{* *}$ & $44.4^{* *}$ & $39.3^{* *}$ & 9.3 & $-0.61^{* *}$ & $-35.8^{* *}$ & -16.6 \\
\hline $1 \times 10$ & 2.5 & -0.24 & $-23.1^{* *}$ & $26.9 *$ & 5.0 & -0.36 & -6.2 & 7.2 & 9.4 & $-1.4^{* *}$ & $-34.8^{* *}$ & -28.5 * \\
\hline $2 \times 3$ & 1.5 & $-0.5^{* *}$ & $-33.3^{* *}$ & -22.3 & 3.3 & -0.08 & -0.15 & $-28.6 *$ & 7.3 & $0.86^{* *}$ & $-33.6^{* *}$ & $-40.4^{* *}$ \\
\hline $2 \times 4$ & 2.6 & 0.29 & 16.3 & $34.7^{* *}$ & 5.0 & 0.63 & -21.07 * & 7.2 & 7.8 & 0.31 & $-29.1^{* *}$ & $-33.3 *$ \\
\hline $2 \times 5$ & 2.1 & 0.05 & -6.04 & 8.81 & 3.3 & -0.05 & 11 & $-28.6^{*}$ & 7.6 & $0.85^{* *}$ & $-30.4^{* *}$ & $-38.1^{* *}$ \\
\hline $2 \times 6$ & 2.6 & 0.15 & 0.76 & $36.5^{* *}$ & 6.2 & $1.0^{* *}$ & -11.9 & $32.1 *$ & 9.3 & -0.009 & $-15.0 * *$ & $-50.0^{* *}$ \\
\hline $2 \times 7$ & 2.2 & -0.19 & -5.7 & 11.4 & 5.0 & 0.35 & 11.1 & 7.1 & 8.9 & $0.72 * *$ & $-18.6^{* *}$ & $-52.3^{* *}$ \\
\hline $2 \times 8$ & 2.7 & 0.18 & -6.8 & $38.3^{* *}$ & 5.0 & 0.46 & -14.2 & 7.1 & 8.7 & -0.15 & $-20.9^{* *}$ & $-45.2^{* *}$ \\
\hline $2 \times 9$ & 2.1 & -0.15 & -11.2 & 8.03 & 3.8 & -0.35 & -11.5 & -17.9 & 8.5 & $-0.72 * *$ & $-22.7 * *$ & -23.8 \\
\hline $2 \times 10$ & 2.6 & -0.008 & $-18.4 *$ & $34.7^{* *}$ & 3.3 & $-1.0 * *$ & $-37.5^{* *}$ & -28.6 * & 7.5 & $-2.6^{* *}$ & $-31.8 * *$ & -2.3 \\
\hline $3 \times 4$ & 2.4 & 0.14 & 7.3 & 25.1 & 3.7 & -0.41 & $-42.1^{* *}$ & -21.4 & 4.6 & -0.03 & $-41.1 * *$ & 14.2 \\
\hline $3 \times 5$ & 1.9 & -0.07 & -17.1 & -3.3 & 3.7 & 0.57 & 10.0 & -21.3 & 5.2 & $1.2^{* *}$ & $-11.1^{*}$ & $-38.1^{* *}$ \\
\hline $3 \times 6$ & 2.5 & 0.07 & -6.3 & $26.9 *$ & 4.3 & -0.52 & $-38.0 * *$ & -7.07 & 4.8 & $-1.7^{* *}$ & $-54.4^{* *}$ & $45.2^{* *}$ \\
\hline $3 \times 7$ & 2.4 & 0.1 & 5.3 & 24.3 & 3.7 & -0.68 & -18.4 & -21.3 & 4. & $-1.2^{* *}$ & $-52.2 * *$ & -16.6 \\
\hline $3 \times 8$ & 2.5 & 0.1 & -11.5 & 31.3 * & 2.5 & $-1.7^{* *}$ & $-57.1^{* *}$ & $-46.4^{* *}$ & 5.3 & $-0.75^{* *}$ & $-15.8^{* *}$ & -16.6 \\
\hline $3 \times 9$ & 1.9 & -0.2 & -19.1 & -1.5 & 4.7 & $0.76^{*}$ & 7.7 & 0.0 & 6.0 & $-0.42 *$ & 2.5 & -7.1 \\
\hline $3 \times 10$ & 2.5 & -0.05 & $-23.1^{* *}$ & $26.9 *$ & 5.0 & $0.87 *$ & -6.2 & 7.18 & 5. & $-1.9 * *$ & $-49.3^{* *}$ & $-30.9 *$ \\
\hline $4 \times 5$ & 2.2 & -0.04 & -1.3 & 13.9 & 3.2 & $-0.88 *$ & $-50.0^{* *}$ & $-32.1 *$ & 6. & $1.09^{* *}$ & $-22.1^{* *}$ & $-26.2 *$ \\
\hline $4 \times 6$ & 3.4 & $0.6^{*}$ & $28.6^{* *}$ & $74.3^{* *}$ & 5.8 & 0.01 & -16.7 * & $24.9 *$ & 5. & -2.0 ** & $-47.9^{* *}$ & $-40.4^{* *}$ \\
\hline $4 \times 7$ & 2.3 & -0.21 & 2.1 & 20.7 & 5.5 & 0.18 & -13.2 & 17.9 & 5. & $-0.6^{* *}$ & $-34.2 * *$ & -9.5 \\
\hline $4 \times 8$ & 2.9 & 0.18 & 0.17 & $48.7^{* *}$ & & 0.2 & -13.2 & 17.9 & 7. & $0.59 * *$ & -2.5 & $-38.1^{* *}$ \\
\hline $4 \times 9$ & 2.2 & -0.19 & -5.7 & 14.7 & 3.8 & $-1.0 * *$ & $-39.4^{* *}$ & -17.7 & 5.9 & $-1.5^{* *}$ & $-25.3 * *$ & $-38.1^{* *}$ \\
\hline $4 \times 10$ & 2.8 & -0.05 & -13.6 & $42.5^{* *}$ & 4.2 & $-0.91 *$ & $-34.2^{* *}$ & -10.6 & 5.5 & $-2.8^{* *}$ & $-48.3^{* *}$ & $-54.7^{* * *}$ \\
\hline $5 \times 6$ & 2.6 & 0.26 & 0.0 & $35.5^{* *}$ & 6. & $1.1^{* *}$ & -14.7 & $27.8 *$ & 5. & $-1.1^{* *}$ & $-46.0 * *$ & $-40.4^{* *}$ \\
\hline $5 \times 7$ & 2.3 & 0.1 & 1.5 & 19.9 & 3.7 & -0.66 & -18.5 & -21.4 & 5.5 & -0.29 & $-38.2 * *$ & -16.6 \\
\hline $5 \times 8$ & 2.2 & -0.12 & $-21.2 *$ & 15.8 & 4.2 & -0.04 & $-28.5^{* *}$ & -10.7 & 5.3 & $-1.0 * *$ & $-15.1^{* *}$ & $-54.7^{* * *}$ \\
\hline $5 \times 9$ & 2.0 & -0.13 & -17.0 & 1.04 & 4.0 & 0.13 & -7.6 & -14.2 & 5. & $-1.6^{* *}$ & -11.9 * & -16.6 \\
\hline $5 \times 10$ & 2.3 & -0.23 & $-29.3^{* *}$ & 16.5 & 4. & -0.09 & $-25.0 *$ & -14.2 & 6. & $-1.3^{* *}$ & $-40.9^{* *}$ & -26.2 * \\
\hline $6 \times 7$ & 2.5 & -0.13 & -3.82 & 30.3 * & 5. & $-1.0 * *$ & $-28.5^{* *}$ & 7.1 & 7. & -0.40 * & $-25.3 * *$ & $-26.2 *$ \\
\hline $6 \times 8$ & 2.7 & -0.12 & -6.81 & $38.3^{* *}$ & 5. & -0.31 & -19.0 * & 21.4 & 7. & $-1.5^{* *}$ & $-30.0^{* *}$ & $-45.2^{* *}$ \\
\hline $6 \times 9$ & 2.0 & $-0.4^{* *}$ & -22.7 * & 4.6 & 4.0 & $-1.6^{* *}$ & $-42.8^{* *}$ & -14.2 & 13.7 & $4.4^{* *}$ & $29.1^{* *}$ & $-47.6^{* *}$ \\
\hline $6 \times 10$ & 2.6 & -0.3 * & -18.8 * & $33.9^{* *}$ & 5.5 & -0.35 & $-21.4 *$ & 17.9 & 10.9 & $0.68^{* *}$ & 1.8 & $-57.1^{* *}$ \\
\hline $7 \times 8$ & 2.6 & -0.0 & -8.7 & $35.5 * *$ & 6.8 & 1.3 & 16.3 & $45.4^{* *}$ & 7. & $-0.5^{* *}$ & $-17.9^{* *}$ & $-45.2^{* *}$ \\
\hline $7 \times 9$ & 2.6 & 0.18 & 9.1 & $32.9 *$ & 6.3 & 1.2 ** & $40.7^{* *}$ & $35.8^{* *}$ & 8.9 & 0.68 ** & 0.0 & -26.2 * \\
\hline $7 \times 10$ & 3.0 & 0.2 & -6.4 & $54.4^{* *}$ & 6.7 & $1.3^{* *}$ & $25.0 *$ & $42.8^{* *}$ & 8.6 & $-0.4^{* *}$ & $-19.5^{* *}$ & $-71.4^{* *}$ \\
\hline $8 \times 9$ & 2.4 & -0.09 & -15.0 & $26.2 *$ & 5.0 & -0.02 & -14.2 & 7.2 & 11 & $2.8^{* *}$ & $85.7^{* *}$ & $-38.1^{* *}$ \\
\hline $8 \times 10$ & 2.9 & -0.01 & -8.4 & $51.0^{* *}$ & 5.2 & -0.07 & -11.3 & 10.8 & 16.5 & $6.7^{* *}$ & $53.4^{* *}$ & $-45.2^{* *}$ \\
\hline $9 \times 10$ & 3.1 & $0.4^{* *}$ & -2.6 & $60.6^{* *}$ & 5.2 & 0.26 & -3.1 & 10.8 & 13.9 & $3.7^{* *}$ & $29.3^{* *}$ & -33.3 * \\
\hline $\begin{array}{l}\text { LSD } \\
(p \leq 0.05)\end{array}$ & 0.48 & 0.31 & 0.49 & 0.49 & 1.1 & 0.75 & 1.1 & 1.2 & 0.59 & 0.35 & 0.54 & 0.05 \\
\hline
\end{tabular}

* Significant at the $5 \%$ level; ${ }^{* *}$ significant at the $1 \%$ level. Abbreviation: SCA-specific combining ability; $\mathrm{BPH}$ - better parent heterosis; $\mathrm{SDH}$-standard heterosis.

For rind thickness, the highest positive and negative GCA values were exhibited by MM-625 (0.90) and MM-904 (-0.86), respectively (Table 3). The parental line MM-625 also exhibited the highest rind thickness $(7.0 \mathrm{~mm})$, while the thinnest rind was observed in 
Kajri $(2.9 \mathrm{~mm})$. For rind thickness, the highest SCA effect was observed in MS- $1 \times$ MM 916 (Table 5). This cross also exhibited 44.4\% BPH over MS-1 (Table 5). Meanwhile, the highest SDH was observed in the cross MS- $\times$ MM-625 (50.0\%) (Table 5, Figure 2). Besides this, the MM-610 $\times$ MM-1831, MM-610 $\times$ MM-916 and MM-610 $\times$ Riogold hybrids also exhibited a significant SDH for rind thickness (Table 5).

The mean firmness of the hybrids varied from 4.6 to $16.5 \mathrm{lb} / \mathrm{inch}^{2}$, while that of parents varied from 5.8 to $14.5 \mathrm{lb} / \mathrm{inch}^{2}$ (Tables 3 and 5). Among the parental lines, the highest firmness was recorded in MS-1, followed by Kajri, Riogold and MM-625, while the lowest firmness was observed in $\mathrm{KP}_{4} \mathrm{HM}-15$. The GCA effect for firmness ranged from -2.1 to 1.5 (Table 3). The highest positive GCA was exhibited by Riogold, and the lowest negative GCA was calculated for $\mathrm{KP}_{4} \mathrm{HM}-15$. For firmness, the highest SCA effect was observed in MM-1831 $\times$ Riogold (Table 5, Figure 3). This cross also exhibited 53.4\% BPH over Riogold (Table 5). Besides this, the MM- $1831 \times$ MM-916 hybrid also exhibited a significant $\mathrm{BPH}$ for this trait. Meanwhile, the cross $\mathrm{KP}_{4} \mathrm{HM}-15 \times \mathrm{MM}-625$ exhibited the maximum positive $\mathrm{SDH}$ (45.2\%).

The highest positive and negative GCA values for total soluble solids were recorded in MM 916 and Kajri, respectively (Table 3). The maximum SCA effect for total soluble solids was observed in hybrid MS- $1 \times$ Kajri, followed by Kajri $\times$ MM- $904, \mathrm{KP}_{4} \mathrm{HM}-15 \times \mathrm{MM}-103$ and MM-1831 $\times$ MM-916 (Table 6). Hybrid MS-1 $\times$ Kajri also exhibited the higher BPH over MS-1. Meanwhile, for SDH, the highest value was exhibited by hybrid MM- $1831 \times$ MM 916 (44.3\%) over $\mathrm{MH}-27$.

The $\beta$-carotene content of the hybrids varied from 0.21 to $3.4(\mathrm{mg} / 100 \mathrm{~g})$, while that of the parents varied from 0.52 to 2.5 (mg/100 g) (Tables 3 and 6). Although the highest positive GCA value for $\beta$-carotene was recorded in MM- 625 , the highest $\beta$-carotene content was recorded in MM-610, followed by Riogold and MM-625. The hybrid Kajri $\times$ MM-625 exhibited the highest SCA effect, and also exhibited the highest BPH (109.1\%) over MM-625. Hybrid MM-610 $\times$ MM-1831 (9.2\%) exhibited the highest SDH, followed by MS- $1 \times$ MM-1831 $(6.5 \%)$.

The highest positive and negative GCA values for ascorbic acid were exhibited by MM-610 and MM Sel-103, respectively (Table 3). The maximum SCA effect for ascorbic acid content was observed in hybrid MS-1 $\times$ MM Sel-103 (Table 6). No hybrid exhibited a positive significant BPH for this trait. Meanwhile, the cross Kajri $\times$ MM-625 (63.3\%) exhibited the maximum and positive SDH over the commercial check, MH-27 (Table 6). In addition, hybrids MM-610 $\times$ MM-916, MM-1831 $\times$ Riogold and MM-916 $\times$ Riogold were also found to be promising for this trait (Figures 2 and 3).

\subsection{Disease Reaction}

The reaction to Fusarium wilt incidence of the hybrids varied from 12.0 to 40.5 PDI, while that of the parents varied from 19.5 to 38.5 PDI (Tables 3 and 6). Highly negative and significant GCA and SCA effects were considered for the disease-resistant cultivars. The significant result revealed that the disease incidence was controlled by both additive and non-additive gene action (Table 2). For the reaction to Fusarium wilt, the highest negative GCA value was recorded in inbred MM-610 (-3.5), followed by MM Sel-103 (-2.8) and Kajri (-2.3) (Table 3). The highest positive GCA effect was exhibited by MM-916 (2.4). However, MM-610 and MM-916 exhibited contrasting symptoms of wilt infestation, with MM-610 being moderately susceptible, while MM-916 was resistant. The most favorable SCA effect was observed in MM Sel-103 $\times$ MM-625 (-17.3). Cross MS- $1 \times$ Kajri exhibited the highest BPH over Kajri (-67.3\%) and the second highest SDH $(-63.2 \%)$ among all of the 45 hybrids. As inbred Kajri had resistance to Fusarium wilt, the cross derived from it also displayed resistant symptoms. Furthermore, the cross MM Sel-103 $\times$ MM- 625 had the highest resistance to wilt, and showed the greatest negative economic heterosis over MH-27 (-64.7\%) (Table 7). 
Table 6. Estimation of the mean, specific combining ability, better parent and standard heterosis (\%) over the commercial check MH-27 for TSS, $\beta$-carotene and ascorbic acid of muskmelon.

\begin{tabular}{|c|c|c|c|c|c|c|c|c|c|c|c|c|}
\hline \multirow{2}{*}{ Hybrid } & \multicolumn{4}{|c|}{ TSS ( ${ }^{\circ}$ Brix) } & \multicolumn{4}{|c|}{$\beta$-Carotene (mg/100 g) } & \multicolumn{4}{|c|}{ Ascorbic Acid (mg/100 g) } \\
\hline & Mean & SCA & ВPH & SDH & Mean & SCA & ВРH & $\mathrm{SDH}$ & Mean & SCA & ВРH & $\mathrm{SDH}$ \\
\hline $1 \times 2$ & 13.1 & $3.2 * *$ & $50.7 * *$ & $27.3^{* *}$ & 1.2 & $-0.15^{* *}$ & $-27.8^{* *}$ & $-20.7^{* *}$ & 19.5 & $-1.4^{*}$ & $-25.8^{* *}$ & $-42.1^{* *}$ \\
\hline $1 \times 3$ & 12.0 & 1.0 & 7.5 & $16.5^{*}$ & 1.7 & $0.49^{* *}$ & 0.89 & $-48.1^{* *}$ & 12.7 & $-5.5^{* *}$ & $-51.4^{* *}$ & $-19.0^{* *}$ \\
\hline $1 \times 4$ & 10.6 & 0.07 & 16.5 & 3.3 & 1.5 & $-0.17 * *$ & $-12.9 * *$ & -1.4 & 24.2 & $6.5^{* *}$ & $-7.7 *$ & $-27.8^{* *}$ \\
\hline $1 \times 5$ & 10.6 & 0.5 & 18.0 & 3.1 & 1.1 & 0.001 & $-35.3^{* *}$ & -7.5 & 22.7 & $1.6^{* *}$ & $-13.4^{* *}$ & $-48.1^{* *}$ \\
\hline $1 \times 6$ & 10.4 & -0.34 & 0.48 & 1.2 & 2.3 & $0.21 * *$ & $37.9 * *$ & $-56.2^{* *}$ & 10.7 & $-0.6^{* *}$ & $-60.4^{* *}$ & $10.7^{* *}$ \\
\hline $1 \times 7$ & 10.5 & 0.04 & 2.23 & 2.2 & 1.9 & 0.1 & $-22.3^{* *}$ & -5.9 & 23.1 & 0.92 & $-12.7^{* *}$ & -6.6 \\
\hline $1 \times 8$ & 11.0 & -0.25 & 5.6 & 6.4 & 2.1 & $0.49 * *$ & $27.6^{* *}$ & 6.5 & 26.2 & $5.4^{* *}$ & -0.3 & 2.3 \\
\hline $1 \times 9$ & 11.5 & -0.15 & $28.3^{* *}$ & 11.7 & 1.5 & $-0.2 * *$ & -7.4 & $-13.3^{* *}$ & 21.3 & 0.52 & $-18.9^{* *}$ & $-25.7^{* *}$ \\
\hline $1 \times 10$ & 9.3 & -0.99 & 2.5 & -9.3 & 1.0 & $-0.8^{* *}$ & $-56.4^{* *}$ & $-49.1^{* *}$ & 12.5 & $-6.3^{* *}$ & $-52.4^{* *}$ & $-52.1^{* *}$ \\
\hline $2 \times 3$ & 6.5 & $-4.0 * *$ & $-41.6^{* *}$ & $-36.7^{* *}$ & 0.49 & $-0.4^{* *}$ & -22.4 & $-27.6^{* *}$ & 17.8 & 0.45 & $-15.9^{* *}$ & $-76.9^{* *}$ \\
\hline $2 \times 4$ & 10.7 & 0.53 & 16.6 & 3.5 & 1.9 & $0.47^{* *}$ & $10.6^{*}$ & $-27.0 * *$ & 17.9 & $1.2^{*}$ & $-15.2^{* *}$ & $-8.3^{*}$ \\
\hline $2 \times 5$ & 12.2 & $2.4^{* *}$ & $35.0 * *$ & 18.0 * & 0.21 & $-0.6^{* *}$ & $-61.4^{* *}$ & $-43.8^{* *}$ & 13.8 & $-6.3^{* *}$ & $-45.6^{* *}$ & $-90.0^{* *}$ \\
\hline $2 \times 6$ & 9.5 & -0.83 & -8.5 & -7.7 & 3.4 & $1.56^{* *}$ & $109.1^{* *}$ & $-22.2^{* *}$ & 19.1 & $-1.3^{*}$ & $-29.6^{* *}$ & $63.3^{* *}$ \\
\hline $2 \times 7$ & 9.3 & -0.72 & -9.5 & -9.5 & 0.45 & $-1.1^{* *}$ & $-82.3^{* *}$ & $-9.8 *$ & 22.2 & 0.92 & $-16.3^{* *}$ & $-78.8^{* *}$ \\
\hline $2 \times 8$ & 11.8 & 1.0 & 14.1 & 15.0 & 2.1 & $0.68^{* *}$ & $39.3^{* *}$ & $-16.0 * *$ & 20.6 & 0.88 & $-12.7^{* *}$ & -0.48 \\
\hline $2 \times 9$ & 12.8 & $1.5^{* *}$ & $42.5^{* *}$ & $24.1^{* *}$ & 2.3 & 0.72 ** & $58.2^{* *}$ & -6.2 & 23.0 & $3.2^{* *}$ & 6.4 & $9.2 *$ \\
\hline $2 \times 10$ & 11.7 & $1.8^{* *}$ & $28.8 * *$ & 13.8 & 1.7 & $0.15 * *$ & $-22.9 * *$ & $-26.9^{* *}$ & 17.9 & 0.03 & $-15.1^{* *}$ & $-15.2 * *$ \\
\hline $3 \times 4$ & 13.5 & $2.2 * *$ & $20.5^{* *}$ & $30.6^{* *}$ & 1.9 & 0.62 ** & $9.7 *$ & $-28.7^{* *}$ & 17.5 & $3.4^{* *}$ & -1.6 & $-9.1 *$ \\
\hline $3 \times 5$ & 8.8 & $-2.0 * *$ & $-21.1^{* *}$ & -14.5 & 0.6 & -0.09 & -4.8 & $-12.2^{* *}$ & 21.6 & $4.1^{* *}$ & $-14.8^{* *}$ & $-71.6^{* *}$ \\
\hline $3 \times 6$ & 11.8 & 0.32 & 5.5 & 14.3 & 2.1 & $0.37 * *$ & $26.8^{* *}$ & $-10.5^{* *}$ & 22.0 & $4.2^{* *}$ & $-19.1^{* *}$ & -0.95 \\
\hline $3 \times 7$ & 11.6 & 0.43 & 3.8 & 12.5 & 1.2 & $-0.25^{* *}$ & $-52.6^{* *}$ & $-47.1^{* *}$ & 13.0 & $-5.6^{* *}$ & $-50.9^{* *}$ & $-43.1^{* *}$ \\
\hline $3 \times 8$ & 13.2 & $1.3 *$ & $18.3 *$ & $28.2 * *$ & 0.75 & $-0.50 * *$ & $-50.3^{* *}$ & $-62.1^{* *}$ & 9.33 & $-7.8^{* *}$ & $-60.5^{* *}$ & $-64.5^{* *}$ \\
\hline $3 \times 9$ & 13.0 & 0.64 & 16.3 * & $26.1^{* *}$ & 1.3 & -0.03 & -4.8 & $-14.6^{* *}$ & 20.9 & $3.7^{* *}$ & -3.1 & $-34.3^{* *}$ \\
\hline $3 \times 10$ & 12.1 & 1.0 & 8.0 & $17.1^{*}$ & 1.7 & $0.23^{* *}$ & $-26.2^{* *}$ & $-50.3^{* *}$ & 12.2 & $-3.2^{* *}$ & $-35.8^{* *}$ & $-18.8^{* *}$ \\
\hline $4 \times 5$ & 10.9 & 0.5 & $19.2 *$ & 5.8 & 0.78 & $-0.39 * *$ & $-55.4^{* *}$ & $-14.3^{* *}$ & 21.2 & $4.1^{* *}$ & $-16.9^{* *}$ & $-63.1^{* *}$ \\
\hline $4 \times 6$ & 11.8 & 0.7 & 13.6 & 14.5 & 2.3 & 0.1 & $31.9 * *$ & $-50.1^{* *}$ & 12.2 & $-4.9^{* *}$ & $-54.8^{* *}$ & $9.2 *$ \\
\hline $4 \times 7$ & 9.5 & -1.2 * & -7.7 & -7.7 & 2.1 & $0.22 * *$ & $-14.4^{* *}$ & $-31.8^{* *}$ & 16.7 & $-1.2 *$ & $-36.7^{* *}$ & 2.8 \\
\hline $4 \times 8$ & 10.2 & -1.3 * & -1.8 & -1.1 & 0.95 & $-0.7^{* *}$ & $-45.4^{* *}$ & $-52.5^{* *}$ & 11.6 & $-4.8^{* *}$ & $-50.7^{* *}$ & $-54.7^{* *}$ \\
\hline $4 \times 9$ & 13.6 & $1.6^{* *}$ & $48.6^{* *}$ & $31.9^{* *}$ & 2.6 & $0.69 * *$ & $49.1^{* *}$ & $-28.2 * *$ & 17.6 & 1.0 & $-18.4^{* *}$ & $23.5 * *$ \\
\hline $4 \times 10$ & 10.9 & 0.26 & $18.8^{*}$ & 5.4 & 1.2 & $-0.7^{* *}$ & $-46.3^{* *}$ & $-48.5^{* *}$ & 12.6 & $-2.0 * *$ & $-33.5^{* *}$ & $-40.9^{* *}$ \\
\hline $5 \times 6$ & 12.6 & $1.9^{* *}$ & 21.1 * & $22.1 * *$ & 2.9 & $1.31^{* *}$ & $77.1^{* *}$ & $-14.6^{* *}$ & 20.9 & 0.35 & $-22.8^{* *}$ & $38.3^{* *}$ \\
\hline $5 \times 7$ & 9.62 & -0.72 & -6.9 & -6.9 & 0.61 & $-0.7^{* *}$ & $-75.8^{* *}$ & $-40.3^{* *}$ & 14.6 & $-6.7^{* *}$ & $-44.6^{* *}$ & $-70.9 * *$ \\
\hline $5 \times 8$ & 10.9 & -0.12 & 5.1 & 5.9 & 1.3 & $0.24 * *$ & -8.3 & $-36.3^{* *}$ & 15.6 & $-4.2^{* *}$ & $-38.2 * *$ & $-34.5^{* *}$ \\
\hline $5 \times 9$ & 12.7 & $1.2 *$ & $41.4^{* *}$ & $23.6^{* *}$ & 0.99 & $-0.3^{* *}$ & $-32.2^{* *}$ & $-29.7^{* *}$ & 17.2 & $-2.7^{* *}$ & $-31.9^{* *}$ & $-53.1^{* *}$ \\
\hline $5 \times 10$ & 8.2 & $-1.9^{* *}$ & -10.2 & $-20.6^{*}$ & 2.0 & $0.6^{* *}$ & $-11.6^{* *}$ & $-26.2^{* *}$ & 18.1 & 0.01 & $-28.5^{* *}$ & -2.8 \\
\hline $6 \times 7$ & 12.0 & 1.0 & 15.2 & 16.1 & 2.3 & -0.01 & $-7.1^{*}$ & -6.5 & 22.9 & $1.2 *$ & $-15.4^{* *}$ & $11.6^{* *}$ \\
\hline $6 \times 8$ & 11.2 & -0.56 & 7.2 & 8.0 & 2.0 & $-0.1^{*}$ & $24.1^{* *}$ & -3.4 & 23.7 & $3.5^{* *}$ & $-12.7 * *$ & -3.1 \\
\hline $6 \times 9$ & 11.8 & -0.36 & 13.4 & 14.3 & 1.7 & $-0.5^{* *}$ & 7.3 & $-43.8^{* *}$ & 13.8 & $-6.5^{* *}$ & $-49.2 * *$ & $-16.2 * *$ \\
\hline $6 \times 10$ & 10.6 & -0.24 & 1.6 & 2.4 & 1.4 & $-0.9 * *$ & $-37.6^{* *}$ & $-19.4^{* *}$ & 19.8 & $1.4^{*}$ & $-27.1 * *$ & $-31.4^{* *}$ \\
\hline $7 \times 8$ & 11.1 & -0.37 & 6.2 & 7.0 & 2.2 & $0.3^{* *}$ & $-12.8^{* *}$ & $9.2 *$ & 26.8 & $5.7^{* *}$ & 1.2 & 4.7 \\
\hline $7 \times 9$ & 13.2 & $1.2 *$ & $27.3^{* *}$ & $27.3^{* *}$ & 3.0 & $0.9^{* *}$ & $19.4^{* *}$ & $-26.1^{* *}$ & 18.1 & $-2.9^{* *}$ & $-31.5^{* *}$ & $43.5^{* *}$ \\
\hline $7 \times 10$ & 11.6 & 1.0 & 12.2 & 12.2 & 1.8 & $-0.2^{* *}$ & $-27.1^{* *}$ & $-22.7^{* *}$ & 19.0 & -0.21 & $-28.3^{* *}$ & $-12.3^{* *}$ \\
\hline $8 \times 9$ & 14.9 & $2.2^{* *}$ & $43.2 * *$ & $44.3^{* *}$ & 1.2 & $-0.6^{* *}$ & $-21.6^{* *}$ & $-42.8^{* *}$ & 14.0 & $-5.5^{* *}$ & $-40.6^{* *}$ & $-44.0^{* *}$ \\
\hline $8 \times 10$ & 12.7 & $1.4^{* *}$ & $22.4^{* *}$ & $23.3^{* *}$ & 2.6 & $0.76^{* *}$ & $15.8^{* *}$ & $-33.6^{* *}$ & 16.3 & $-1.3^{*}$ & $-31.0^{* *}$ & $27.3^{* *}$ \\
\hline $9 \times 10$ & 11.7 & 0.01 & $28.7 * *$ & 13.6 & 2.7 & $0.6^{* *}$ & $16.2^{* *}$ & -6.2 & 23.0 & $5.2^{* *}$ & 6.3 & $27.8^{* *}$ \\
\hline $\begin{array}{c}\text { LSD } \\
(p \leq 0.05)\end{array}$ & 1.6 & 1.1 & 1.7 & 1.7 & 0.16 & 0.1 & 0.17 & 1.8 & 1.8 & 1.2 & 1.9 & 0.16 \\
\hline
\end{tabular}


Table 7. Estimation of the percent disease index, specific combining ability, better parent and standard heterosis (\%) over the commercial check MH-27 for the reaction to Fusarium wilt of muskmelon.

\begin{tabular}{|c|c|c|c|c|c|c|c|c|c|c|c|}
\hline Hybrid & PDI & SCA & ВPH & SDH & Reaction & Hybrid & PDI & SCA & ВРH & SDH & Reaction \\
\hline $1 \times 2$ & 12.5 & $-6.1^{* *}$ & $-67.3^{* *}$ & $-63.2^{* *}$ & Resistant & $3 \times 10$ & 35.1 & 0.99 & 1.9 & 3.4 & Moderately Resistant \\
\hline $1 \times 3$ & 33.9 & $1.6^{* *}$ & $-5.8 *$ & -0.29 & Moderately Resistant & $4 \times 5$ & 20.5 & $-9.7 * *$ & $-45.4^{* *}$ & $-39.7^{* *}$ & Resistant \\
\hline $1 \times 4$ & 24.0 & $-4.1^{* *}$ & $-33.3^{* *}$ & $-29.4^{* *}$ & Resistant & $4 \times 6$ & 12.0 & $-17.3^{* *}$ & $-66.6^{* *}$ & $-64.7^{* *}$ & Resistant \\
\hline $1 \times 6$ & 33.0 & 0.92 & $-8.3^{* *}$ & -2.9 & Moderately Resistant & $4 \times 8$ & 37.0 & $8.5^{* *}$ & 2.9 & $8.8^{* *}$ & Moderately Resistant \\
\hline $1 \times 7$ & 28.5 & 1.1 & $-20.8^{* *}$ & $-16.2^{* *}$ & Resistant & $4 \times 9$ & 35.5 & $4.8^{* *}$ & $-5.7 *$ & 4.4 & Moderately Resistant \\
\hline $1 \times 8$ & 35.7 & $4.5^{* *}$ & -0.76 & 5.1 & Moderately Resistant & $4 \times 10$ & 32.2 & $2.2^{* *}$ & $-10.2^{* *}$ & -5.1 & Moderately Resistant \\
\hline $1 \times 9$ & 31.5 & $-1.8^{* *}$ & $-16.3^{* *}$ & $-7.3^{* *}$ & Moderately Resistant & $5 \times 6$ & 37.5 & $3.3^{* *}$ & 0.03 & $10.4^{* *}$ & Moderately Resistant \\
\hline $2 \times 3$ & 35.5 & $5.3^{* *}$ & $-7.2^{* *}$ & 4.4 & Moderately Resistant & $5 \times 8$ & 36.1 & $2.7 * *$ & -3.9 & $6.0^{*}$ & Moderately Resistant \\
\hline $2 \times 4$ & 20.0 & $-5.9 * *$ & $-47.7^{* *}$ & $-41.2^{* *}$ & Resistant & $5 \times 9$ & 40.5 & $4.9^{* *}$ & $7.5^{* *}$ & $19.1^{* *}$ & Moderately Resistant \\
\hline $2 \times 5$ & 16.0 & $-14.8^{* *}$ & $-58.2 * *$ & $-52.9^{* *}$ & Resistant & $5 \times 10$ & 37.0 & $2.1 * *$ & -1.4 & $8.8^{* *}$ & Moderately Resistant \\
\hline $2 \times 6$ & 35.5 & $5.5^{* *}$ & $-7.2 * *$ & 4.4 & Moderately Resistant & $6 \times 7$ & 36.0 & $7.3^{* *}$ & $6.9 *$ & $5.8 *$ & Moderately Resistant \\
\hline $2 \times 7$ & 27.5 & $2.3^{* *}$ & $-28.1^{* *}$ & $-19.1^{* *}$ & Resistant & $6 \times 8$ & 27.0 & $-5.4^{* *}$ & $-19.7^{* *}$ & $-20.6^{* *}$ & Resistant \\
\hline $2 \times 8$ & 27.5 & $-1.4^{*}$ & $-28.1^{* *}$ & $-19.1^{* *}$ & Resistant & $6 \times 9$ & 38.5 & $3.8^{* *}$ & 2.2 & $13.2^{* *}$ & Moderately Resistant \\
\hline $2 \times 9$ & 31.0 & -0.17 & $-19.0^{* *}$ & $-8.8^{* *}$ & Moderately Resistant & $6 \times 10$ & 36.2 & $2.3^{* *}$ & 5.1 & $6.6^{*}$ & Moderately Resistant \\
\hline $2 \times 10$ & 32.0 & $1.5 *$ & $-16.4^{* *}$ & $-5.8 *$ & Moderately Resistant & $7 \times 8$ & 35.5 & $7.8^{* *}$ & $54.3 * *$ & 4.4 & Moderately Resistant \\
\hline $3 \times 4$ & 35.2 & $5.6^{* *}$ & -1.9 & 3.5 & Moderately Resistant & $7 \times 9$ & 20.0 & $-9.9^{* *}$ & $-46.8^{* *}$ & $-41.2^{* *}$ & Resistant \\
\hline $3 \times 6$ & 32.5 & -1.08 & -3.4 & -4.4 & Moderately Resistant & $8 \times 9$ & 29.0 & $-4.6^{* *}$ & $-22.9 * *$ & $-14.7 * *$ & Resistant \\
\hline $3 \times 7$ & 36.1 & $7.2^{* *}$ & $53.6^{* *}$ & $6.2^{*}$ & Moderately Resistant & $8 \times 10$ & 35.5 & $2.5^{* *}$ & 2.9 & 4.4 & Moderately Resistant \\
\hline $3 \times 8$ & 35.0 & $2.3^{* *}$ & $48.9^{* *}$ & 2.9 & Moderately Resistant & $9 \times 10$ & 32.5 & $-2.7^{* *}$ & $-13.6^{* *}$ & -4.4 & Moderately Resistant \\
\hline $3 \times 9$ & 37.0 & $2.1^{* *}$ & -1.7 & $8.8^{* *}$ & Moderately Resistant & $\operatorname{LSD}(p \leq 0.05)$ & 1.8 & 1.5 & 1.8 & 1.8 & \\
\hline
\end{tabular}

* Significant at the $5 \%$ level; ** significant at the $1 \%$ level. Abbreviations: PDI—percent disease index; SCA—specific combining ability; BPH—better parent heterosis; SDH—-standard heterosis. 


\subsection{Genetic Diversity among Ten Parental Lines and Hybrid Performance}

In order to determine parental polymorphism, a total of 121 primers were applied on all ten parental lines. Out of these primers, 70 were found to be polymorphic. These seventy primers were subsequently used to investigate the polymorphism among the parental lines. The pairwise dissimilarity matrix was computed using DARwin 6.0.21 software. This dissimilarity matrix was used to construct a dendrogram by the UPGMA-based neighborjoining tree clustering method. The dissimilarity values ranged from 0.17 to 0.28 , indicating the existence of variability is these ten genotypes (data not shown). The cluster analysis classified the genotypes into three major clusters: Cluster I, Cluster II, and Cluster III (Figure 2). Cluster I, having five genotypes (MM-625, MM-610, MM-1831, MM-916 and Riogold), was further divided into two major sub-clusters: IA and IB. Major sub-cluster IA was further divided into two minor sub-clusters with two genotypes in each. Similarly, Cluster II contained three genotypes (MM Sel-103, MS-1 and Kajri), which were further divided into two major sub-clusters: IIA and IIB. Major sub-cluster IIA was further divided into two minor sub-clusters. Meanwhile, two genotypes (MM-904 andKP $\left.{ }_{4} \mathrm{HM}-15\right)$ were clustered in Cluster III. Hence, the maximum number of genotypes was clustered in Cluster I. In general, Clusters I, II and III included netted, sutured plus netted, and green-fleshed sutured genotypes, respectively. Furthermore, six primers-DM0561, CMAAAGN14, TJ147, CMMS35_3, CMAGN45 and DE1337—revealed unique alleles in five different genotypes; two primers-DM0839 and DE1836 - revealed in two genotypes; CMBR023 identified three genotypes, and DM0214 identified only two cultivars. These markers can be utilized to distinguish cultivars through specific alleles (Table 8).

Table 8. Specific/unique alleles detected by SSR primers, and the identified melon genotypes.

\begin{tabular}{cccc}
\hline Markers & No. of Alleles & Unique Allele(s) & Genotype Identified \\
\hline DM0561 & 4 & 2 & MM Sel-103, MM-610 \\
CMAAAGN14 & 3 & 1 & KP $_{4}$ HM-15, MM-904 \\
TJ147 & 3 & 1 & $\mathrm{KP}_{4}$ HM-15, MM-904 \\
DM0839 & 4 & 1 & MS-1 \\
DE1836 & 4 & 1 & MS-1 \\
CMMS35_3 & 4 & 2 & MM-904, MM-916 \\
CMAGN45 & 3 & 1 & KP $_{4}$ HM-15, MM-916, Riogold \\
DE1337 & 4 & 1 & MM-625, MM-1831 \\
CMBR023 & 3 & 1 & Kajri, MM Sel-103 \\
DM0214 & 4 & 1 & MM-904 \\
\hline
\end{tabular}

\section{Discussion}

\subsection{Yield and Yield Component}

The difference among the entries, including the parental lines and hybrids, were significant for fruit yield and its component traits (Table 2). Tomar et al. [9], Chaudhary et al. [11] and Singh et al. [43] also reported similar results using Indian melon genotypes. The significance of both GCA and SCA variances indicated the role of both additive and nonadditive gene effects in governing these traits. Similar gene actions in controlling the fruit yield, number of fruits per vine and average fruit weight have been reported in previous studies in muskmelon [43-46]. However, Saha et al. [47] and Badami et al. [48] reported the dominant gene action to be responsible for controlling the yield-related traits, such as the fruit weight. The role of additive gene action in controlling the yield per hill, fruits per hill and fruit weight have also been reported previously [49,50]. Feyzian et al. [44] found that two parents-Nahavand and Tashkandi-had a significant positive GCA effect for yield-related traits. Pouyesh et al. [51] also reported that Rish-baba had significant positive GCA effects for total yield, and Ananasi had significant positive GCA effects for fruit weight and the number of fruits per plant.

The ratio of GCA/SCA variances for fruit yield was 0.11 , which differed from the one (1.3) reported by Pouyesh et al. [53]. The lower value of the GCA/SCA ratio indicates 
that the non-additive gene effects were more important in explaining the variation of the fruit yield in this set of parental lines. The significant SCA effects for fruit yield and its components were consistent with the ones reported by Pouyesh et al. [51]. This implied that developing hybrid cultivars could be a promising improvement strategy for yield. The highest magnitude of $\mathrm{BPH}$ for fruit yield was observed in the cross $\mathrm{KP}_{4} \mathrm{HM}-15 \times \mathrm{MM}$ Sel-103. The highest SCA, BPH and SDH for fruit weight was observed in cross MS- $1 \times \mathrm{MM}-$ 610, while the cross MM Sel-103 $\times$ MM-904 had the highest SCA value for the number of fruits per vine (Table 4). Feyzian et al. [44] recorded the highest significant BPH in the crosses Ananasi $\times$ Mashhadi and Abasali $\times$ Hose-sorkh for the average fruit weight and total yield, respectively. Pouyesh et al. [51] reported that the cross Garmak $\times$ Rish-baba was the best specific combiner for the total yield and number of fruits per plant, while the cross Saveh $\times$ Garmak was the best performer for fruit weight. Rolania and Fageria [50] also reported that the cross EC-3 $\times$ GP-211 showed a significant SCA effect with higher fruit weight, fruit yield, and shelf life. Furthermore, Hassan et al. [45] reported that the cross $3 \mathrm{M}-637-\mathrm{D} \times 86 \mathrm{E} 2143$ was derived from low $\times$ high general combiner parents for the total yield per vine, and exhibited the highest SCA effects and highest heterosis for yield.

For fruit yield and its components, the narrow-sense heritability estimates were observed to be low to moderate. The heritabilities for fruit yield, average fruit weight and number of fruits per vine were estimated to be $0.16,0.44$ and 0.18 , respectively. These $h^{2}$ n estimates are also inconsistent with the estimate provided by Kalb and Davis [52]. For fruit number, Zalapa et al. [53] reported heritabilities of 0.45 and 0.28 in two locations.

\subsection{Fruit Morphological Quality Traits}

Flesh thickness, rind thickness and firmness are the main components of fruit quality in muskmelon, and are important for the successful marketability of the fruit $[4,54]$. This study determined the genetic control of both fruit yield and fruit quality traits in Indian, exotic and male sterile melon accessions. The high GCA/SCA variance ratio for flesh thickness and the moderate values for rind thickness and firmness suggested that the genetic improvement of these traits will be easily feasible through selection.

In general, the GCA/SCA variance ratio was greater in the fruit morphological quality traits compared to the yield and its components (Table 2). Similarly, the additive gene action was previously reported for flesh thickness and rind thickness $[15,17,55]$. Contrarily, Badami et al. [48] reported the dominance gene action for fruit flesh thickness.

The exotic cultivar Riogold exhibited the highest mean flesh thickness, as well as a significant GCA for this trait (Table 3). Thus, Riogold can be useful for the further improvement of Indian melons. Considering the importance of additive effects controlling flesh thickness, rind thickness and firmness, the selection for the desired texture and transportability is possible in this population. Barros et al. [15] found that an inbred "Meloa" had a significant GCA effect for flesh thickness.

The hybrids MM Sel-103 $\times$ MM-625, MS-1 $\times$ MM-916, and MM-1831 $\times$ Riogold exhibited the highest SCA effects for flesh thickness, rind thickness, and firmness, respectively. Similarly, Singh et al. [43] reported that the cross combinations MS-5 $\times$ MM-304 and MM-303 $\times$ Punjab sunehri had significant SCA values for flesh thickness and rind thickness, respectively. Furthermore, Feyzian et al. [44] and Costa et al. [17] also observed significant SCA effects and a desirable BPH for flesh thickness. The MM Sel$103 \times$ MM- 625 cross exhibited the highest BPH and SDH over the commercial check, MH-27. Furthermore, Riogold was successful in improving the flesh thickness of the hybrids, MM- $610 \times$ Riogold, MM- $1831 \times$ Riogold, and MM-916 $\times$ Riogold, exhibiting 54.40, 51.04 and $60.62 \% \mathrm{SDH}$, respectively.

\subsection{Biochemical Fruit Quality Traits}

Sweetness (TSS, ${ }^{\circ}$ Brix), $\beta$-carotene and ascorbic acid are the important fruit quality determinants in muskmelon [56]. Similarly, to yield and its component traits, the GCA/SCA variance ratio was low in the fruit biochemical quality traits (Table 2). Therefore, for these 
traits, the contribution of dominance variance was higher than the additive genetic variance (Table 2). These findings were consistent with other studies [46-49]. However, additive gene and both additive and dominant gene actions have also been reported to be predominant in the genetic control of these traits $[15,50,51,57]$. In general, the $h^{2}{ }_{n}$ estimates were low for TSS, $\beta$-carotene and ascorbic acid. Pouyesh et al. [51] reported that the $h^{2}$ estimate for TSS was 0.52 . Thus, a very low proportion of phenotypic variation in these traits is attributable to additive variance.

The Fusarium wilt-resistant line $\mathrm{KP}_{4} \mathrm{HM}-15$ [22] and exotic male sterile line MM 1831 possessing the $m s-3$ gene recorded the highest mean TSS and GCA effect, respectively (Table 3), suggesting that these parental lines can potentially be used to improve the sweetness of Indian muskmelon germplasm. Furthermore, Fusarium wilt resistance is also critical in the improvement of the fruit quality of muskmelon. The cross MS- $1 \times$ Kajri exhibited the highest SCA and BPH (Table 6 and Figure 3). Barros et al. [15] reported that genotype Hy Mark had the significant GCA value for TSS. Among the crosses, AF$646 \times$ Rochedo ranked first for soluble solids. Sedera et al. [57] reported that Fayoum and Beni, and Swif 1 were the best general combiners for TSS and $\beta$-carotene, respectively, and the crosses Aswan $\times$ E1 Behaira and Ananas E1 Dokki $\times$ Beni Swif 1 exhibited the highest SCA values for TSS and $\beta$ carotene, respectively. In the current study, the genotypes MM- 625 and MM-610 were found to be the best general combiners for $\beta$-carotene and ascorbic acid, respectively. The hybrids Kajri $\times$ MM-625 and MM-610 $\times$ MM-1831 exhibited a significant SCA for $\beta$-carotene and ascorbic acid, respectively. Thus, exotic lines MS-1 and MM-1831 can potentially be used to develop improved hybrids with enhanced sweetness, $\beta$-carotene and ascorbic acid. Similarly, Rolania and Fageria [50] identified GP-211 as the best general combiner, and the cross EC-3 $\times$ GP-141 as the best specific combiner for TSS. Singh et al. [43] reported that MM-611, MM-303 and MM-304 were the best general combiners for ascorbic acid content, $\beta$-carotene and TSS. The crosses showing the highest SCA effects for ascorbic acid content, $\beta$ carotene and TSS were MM 2008-8 $\times$ Punjab Sunehri, IC-267375×MM-304 and MS-5×MM 2008-8, respectively.

\subsection{Disease Incidence}

Fusarium wilt (FW), caused by Fusarium oxysporum Schlechtend f. sp. melonis (Leach and Currence) Snyd. and Hans (Fom) is a devastating soil-borne disease of muskmelon which results in serious-to-complete yield losses in susceptible cultivars [5,22]. Numerous crop management approaches, such as crop rotation, soil solarization and grafting have been reported to be ineffective against the FW of melons [5]. Thus, the development of genetically resistant $F_{1}$ hybrids is reckoned as the most appropriate strategy for the management of FW in this crop [4,5,22,31]. Thus, this study utilized the newly evolved FW-resistant inbred lines Kajri, $\mathrm{KP}_{4} \mathrm{HM}-15$ and MM Sel-103 [4,5,22,31] for $\mathrm{F}_{1}$ hybrid development, and to understand the genetic inheritance patterns of FW tolerance for their further utilization.

Both GCA and SCA variances were found to be significant for the reaction to FW, which implies the role of both additive and non-additive gene effects in governing the resistance to FW in melon. Similarly to yield and its component traits, the GCA/SCA variance ratio was low in FW resistance (Table 2). The GCA/SCA variance ratio value for the reaction to FW was 0.09 . Therefore, for this trait, the contribution of dominance variance was higher than the additive genetic variance (Table 2). Moreover, the estimates of $h^{2}{ }_{n}$ was also low. Thus, the genetic improvement for resistance to FW disease is only amenable through the exploitation of heterosis breeding. Perchepied and Pitrat [58] discovered the polygenic inheritance of partial resistance to Fusarium oxysporum f.sp. melonis race 1.2, whereas Herman and Perl-Treves et al. [59] argued that the resistance against three races of Fusarium-0,1 and 2-is controlled by a single dominant gene in melon genotypes. ChikhRouhou et al. [60] found that the inheritance of wilt resistance was governed by three epistatic effects: additive $\times$ additive, dominance $\times$ dominance, and dominance $\times$ additive. Furthermore, studying the Indian germplasm, Deol et al. [5] reported that FW resistance in $\mathrm{KP}_{4} \mathrm{HM}-15$ is governed by a single dominant gene, Fom- $5^{(t)}$. 
The best general combiners for the reaction to FW-MM-610, MM Sel-103 and Kajrican effectively be used to improve the resistance against this pathogen in muskmelon (Table 3). Furthermore, the FW-resistant hybrids MM Sel-103 $\times$ MM-625 and MS- $1 \times$ Kajri were recommended for further evaluation for commercial exploitation. The moderate-tohighly resistant behavior of hybrid combinations involving inbred lines of Indian originMM-610, Kajri, MM Sel-103 and $\mathrm{KP}_{4} \mathrm{HM}-15$-indicated their prospective use in resistance breeding in muskmelon (Table 7). Thus, the resistance genes and unique flavor of Indian landrace inbred lines can effectively be combined with the high $\beta$-carotene and enhanced shelf-life of exotic and/or exotic-derived Indian germplasm for improved $\mathrm{F}_{1}$ hybrids [4].

\subsection{Genetic Diversity among Ten Parental Lines and Hybrid Performance}

Cluster analysis classified the parents into three major clusters-Cluster I, Cluster II, Cluster III (Figure 2) - indicating a fair level of genetic diversity among the parents. The parental lines from Cluster I produced the best-performing $F_{1}$ hybrids when crossed with parental lines from Clusters II and III. Thus, the inter-mating among genotypes from diverse clusters resulted in new recombinants with favorable genes. For example, crosses involving $\mathrm{KP}_{4} \mathrm{HM}-15, \mathrm{MM}$ Sel-103, MM-625, MS-1 and Kajri genotypes belonging to Clusters III, IIB, IB, IIA/2b, and IIA/2a, respectively, produced the best hybrids for yield, flesh thickness, sweetness and Fusarium wilt resistance (Tables 4-7 and Figure 2). Distant crosses would broaden the genetic base, and would help us to breed for high yield and disease resistance through the creation of better recombinant populations.

Several primers revealed unique alleles in different parental lines (Table 8). The polymorphism observed among the parents can be used as a marker for hybrid identification [61].

\section{Conclusions}

The overall aim of the current investigation was to determine the significance of GCA and SCA effects among Indian melons, and to compare these with exotic genotypes in order to develop new $\mathrm{F}_{1}$ hybrids capable of producing high fruit yield with an enhanced level of phytochemicals and shelf-life. This experiment confirmed that Indian landrace-derived genotypes possessing disease resistance can effectively be combined with exotic and/ or exotic-derived melon genotypes for the development of $F_{1}$ hybrids with high fruit yield and enhanced fruit quality. Among 45 hybrids, eight exhibiting more than $50 \%$ heterobeltiosis for fruit yield ha ${ }^{-1}$ along with significant SCA and per se performance were identified to test across environments in order to assess their stability. Male sterility may be transferred to the good combiner genotypes-such as MM Sel-103, KP4HM-15 and MM-625-for the easy and cost-effective production of hybrid seeds. The predominance of non-additive gene action (dominance) in the inheritance of yield and quality components implied that heterosis breeding will be beneficial to obtain accelerated improvements in the muskmelon. The promising hybrids can also provide transgressive segregants in the early segregating generations. The transgressive segregants so generated can be utilized to develop superior inbred lines. Furthermore, the SSR markers can be utilized for the study of the genetic diversity and the identification of the $\mathrm{F}_{1}$ hybrids.

Author Contributions: Conceptualization, S.P.S., N.K.S.; methodology, S.P.S., S.K., N.K.S., R.G., K.A.A.-E., N.C.; validation, S.P.S., S.K., N.K.S., N.C., K.A.A.-E., M.M.H.; formal analysis, S.K., J.K.D.; investigation, S.P.S., S.K., N.K.S.; resources, S.P.S., N.K.S., K.A.A.-E., M.A.A., M.M.H.; writingoriginal draft preparation, S.K. and S.P.S.; writing-review and editing, S.P.S., K.A.A.-E., M.A.A., M.M.H.; visualization, S.P.S., N.K.S.; supervision, S.P.S., K.A.A.-E., M.A.A., M.M.H.; project administration, S.P.S., N.K.S., K.A.A.-E., M.M.H.; funding acquisition, S.P.S., N.K.S., M.M.H. All authors have read and agreed to the published version of the manuscript.

Funding: This research was funded by the Department of Biotechnology, GoI, New Delhi, under an Accelerated Translational Grant for Commercialization (ATGC), grant number BT/PR31469/ATGC/ $127 / 7 / 2019$. Also, the work has been funded by Taif University Researchers Supporting Project number (TURSP-2020/59), Taif University, Taif, Saudi Arabia. 
Institutional Review Board Statement: Not Applicable.

Informed Consent Statement: Not Applicable.

Data Availability Statement: There is no data file other than depicted in the maunscript main text file.

Acknowledgments: The authors are thankful to V.K. Vashisht, Retired Vegetable Breeder, Department of Vegetable Science, PAU, Ludhiana for maintaining the genetic resources used in this study. Furthermore, the authors extend their appreciation to Taif University for supporting the current work through Taif University Researchers Supporting Project number (TURSP-2020/59), Taif University, Taif, Saudi Arabia.

Conflicts of Interest: The authors declare no conflict of interest.

\section{References}

1. Gonzalo, M.J.; Díaz, A.; Dhillon, N.P.S.; Reddy, U.K.; Picó, B.; Monforte, A.J. Re-evaluation of the role of Indian germplasm as center of melon diversification based on genotyping-by-sequencing analysis. BMC Genom. 2019, 20, 1-13. [CrossRef] [PubMed]

2. Stepansky, A.; Kovalski, I.; Perl-Treves, R. Intraspecific classification of melons (Cucumis melo L.) in view of their phenotypic and molecular variation. Plant Syst. Evol. 1999, 217, 313-332. [CrossRef]

3. Fergany, M.; Kaur, B.; Monforte, A.J.; Pitrat, M.; Rys, C.; Lecoq, H.; Dhillon, N.P.S.; Dhaliwal, S.S. Variation in melon (Cucumis melo) landraces adapted to the humid tropics of southern India. Genet. Resour. Crop Evol. 2011, 58, 225-243. [CrossRef]

4. Singh, D.; Leskovar, D.I.; Sharma, S.P.; Sarao, N.K.; Vashisht, V.K. Genetic diversity and interrelationship among Indian and exotic melons based on fruit morphology, quality components and microsatellite markers. Physiol. Mol. Biol. Plants 2020, 26, $985-1002$. [CrossRef] [PubMed]

5. Deol, J.K.; Sharma, S.P.; Rani, R.; Kalia, A.; Chhuneja, P.; Sarao, N.K. Inheritance analysis and identification of SSR markers associated with fusarium wilt resistance in melon. J. Hortic. Sci. Biotechnol. 2021, 14, 1-9. [CrossRef]

6. Pandey, S.; Rai, M.; Prasanna, H.C.; Kalloo, G. "Kashi Madhu”: A new muskmelon cultivar with high total soluble solids. HortScience 2008, 43, 245-246. [CrossRef]

7. Singh, M.; Sharma, S.P.; Sarao, N.K.; Kaur, S.; Chhuneja, P. Molecular mapping of nuclear male-sterility gene ms-1 in muskmelon (Cucumis melo L.). J. Hortic. Sci. Biotechnol. 2020, 95, 162-168. [CrossRef]

8. Rehman, A.; Dang, T.; Qamar, S.; Ilyas, A.; Fatema, R. Revisiting Plant Heterosis_From Field Scale to Molecules. Genes 2021, 12, 1688. [CrossRef]

9. Tomar, R.S.; Bhalala, M.K. Heterosis studies in muskmelon (Cucumis melo L.). J. Hortic. Sci. 2006, 1, $144-147$.

10. Kamer, A.; Yousry, M. Heterosis and heritability studies for fruit characters and yield in melon (Cucumis melo, L.). Middle East J. Appl. Sci. 2015, 5, 262-273.

11. Chaudhary, B.R.; Dhaka, R.S.; Fageria, M.S. Heterosis for yield and yield related attributes in muskmelon (Cucumis melo L.). Indian J. Genet. 2017, 63, 91-92.

12. Munger, H.M. The possible utilization of first generation muskmelon hybrids and an improved method of hybridization. Proc. Am. Soc. Hort. Sci. 1942, 40, 405-410.

13. Griffing, B. Concept of general and specific combining ability in relation to diallel crossing systems. Aust. J. Biol. Sci. 1956, 9, 463 . [CrossRef]

14. Valério, I.P.; Irajá, F.; De Carvalho, F.; De Oliveira, A.C.; De Souza, V.Q. Combining ability of wheat genotypes in two models of diallel analyses. Crop Breed. Appl. Biotechnol. 2009, 9, 100-107. [CrossRef]

15. De Araújo Barros, A.K.; de Sousa Nunes, G.H.; de Queiróz, M.A.; Pereira, E.W.L.; Costa Filho, J.H. Diallel analysis of yield and quality traits of melon fruits. Crop Breed. Appl. Biotechnol. 2011, 11, 313-319. [CrossRef]

16. Hm, S.; Indiresh, K.M.; Lingaiah, H.B. Diallel analysis in brinjal (Solanum melongena L.) for fruit yield, its attributes and bacterial wilt resistance. J. Pharmacogn. Phytochem. 2017, 6, 860-872.

17. Costa, Í.J.N.; de Normandes Valadares, R.; Nóbrega, D.A.; Mendes, A.Q.; Silva, F.S.; Menezes, D. Heterosis and combining ability of melon genotypes of momordica group. J. Exp. Agric. Int. 2019, 30, 1-9. [CrossRef]

18. Nandpuri, K.S.; Singh, S.L.T. Punjab hybrid—a new variety of muskmelon. Progressive farming. agris.fao.org 1982, 18, 3-4.

19. Lal, T.; Vashisht, V.K.; Punjab, D.N. Anmol: A new hybrid of muskmelon (Cucumis melo L.). J. Res. Punjab Agric. Univ. 2007, 44, 83.

20. Kaur, A.; Sharma, M. Comparative performance of muskmelon (Cucumis melo) hybrids at farmers' field in district Kapurthala. J. Krishi Vigyan 2017, 6, 24-31. [CrossRef]

21. Sheng, Y.; Chen, K.; Aolong, F.; Company, A. Genetic diversity of chinese thin-skinned melon cultivars (Cucumis melo L. based on simple sequence repeat markers. Acta Hort. 2007, 763, 169-176. [CrossRef]

22. Kalia, A.; Sharma, S.P.; Vashisht, V.K. Scanning electron microscopy study of root tissue of muskmelon: Transferring fusarium wilt resistance from snapmelon to muskmelon. J. Appl. Nat. Sci. 2017, 9, 1317-1323. [CrossRef]

23. Vashisht, V.K.; Sharma, S.P. MH-51: A new hybrid of muskmelon (Cucumis melo L.). In Proceedings of the 4th National Symposium on Transforming Indian Agriculture Towards Food and Nutritional Security, ICARIGFRI, Jhansi, India, 20-21 February 2016 ; 2016. 
24. Luan, F.; Sheng, Y.; Wang, Y.; Staub, J.E. Performance of melon hybrids derived from parents of diverse geographic Origins. Euphytica 2010, 173, 1-16. [CrossRef]

25. Garcia, E.; Jamilena, M.; Alvarez, J.I.; Arnedo, T.; Oliver, J.L.; Lozano, R. Genetic relationships among melon breeding lines revealed by RAPD markers and agronomic traits. Theor. Appl. Genet. 1998, 96, 878-885. [CrossRef]

26. Mliki, A.; Staub, J.E.; Zhangyong, S.; Ghorbel, A. Genetic diversity in melon (Cucumis melo L.): An evaluation of African germplasm. Genet. Resour. Crop Evol. 2001, 48, 587-597. [CrossRef]

27. Carvalho, N.; Canela, F.M.; Leite, P.H.S.; Ferreira, M.A.; Oliveira, V.R.; Santos, M.F.; Souza, N.O.S.; Buso, G.S.C. Analysis of genetic variability of commercial melon cultivars using SSR molecular markers. Genet. Mol. Res. 2017, 16. [CrossRef] [PubMed]

28. Varshney, R.K.; Graner, A.; Sorrells, M.E. Genic microsatellite markers in plants: Features and applications. Trends Biotechnol. 2005, 23, 48-55. [CrossRef]

29. Staub, J.E.; Danin-Poleg, Y.; Fazio, G.; Horejsi, T.; Reis, N.; Katzir, N. Comparative analysis of cultivated melon groups (Cucumis melo L.) using random amplified polymorphic DNA and simple sequence repeat markers. Euphytica 2000, 115, 225-241. [CrossRef]

30. Pitrat, M. Melon. In Vegetables I. In Handbook of Plant Breeding; Springer: New York, NY, USA, 2008; pp. $283-315$.

31. Vashisht, V.K.; Lal, T.; Sharma, S.P.; Thind, T.S. KP4HM-15: An inbred line resistant to fusarium wilt. In Proceedings of the 3rd International Symposium on Underutilized Plant Species, Madurai, India, 5 August 2015; 2015; pp. 481-486.

32. McCollum, J.P. A rapid method for determining total carotenoids and carotene in tomatoes. Proc. Am. Hort. Sci. 1953, 61, 431-433.

33. Heinze, P.H.; Kanapaux, M.S.; Wade, B.L.; Grimball, P.C.; Foster, R.L.A.A.C. Ascorbic acid content of 39 varieties of snap beans. Food Res. 1944, 9, 19-26. [CrossRef]

34. Zhang, S.P.; Miao, H.; Yang, Y.H.; Xie, B.Y.; Wang, Y.; Gu, X.F. A major quantitative trait locus conferring resistance to fusarium wilt was detected in cucumber by using recombinant inbred lines. Mol. Breed. 2014, 34, 1805-1815. [CrossRef]

35. Bletsos, F.A. Use of grafting and calcium cyanamide as alternatives to methyl bromide soil fumigation and their effects on growth, yield, quality and fusarium wilt control in melon. J. Phytopathol. 2005, 153, 155-161. [CrossRef]

36. Doyle, P.J. DNA Protocols for Plants. In Molecular Techniques in Taxonomy; Hewitt, G.M., Johnaon, A.W.B., Eds.; Springer: Berlin/Heidelberg, Germany; 462 Mann Library, Cornell University: Ithaca, NY, USA, 1991.

37. Voytas, D. Agarose gel electrophoresis. In Current Protocols in Molecular Biology; John Wiley \& Sons: Ames, IA, USA, 2000; pp. 1-9.

38. Zheng, Y.; Wu, S.; Bai, Y.; Sun, H.; Jiao, C.; Guo, S.; Zhao, K.; Blanca, J.; Zhang, Z.; Huang, S.; et al. Cucurbit Genomics Database (CuGenDB): A central portal for comparative and functional genomics of cucurbit crops. Nucleic Acids Res. 2019, 47, D1128-D1136. [CrossRef]

39. Perrier, X.; Jean-Pierre Jacquemoud-Collet. DARwin Software. 2006. Available online: http://darwin.cirad.fr/darwin (accessed on 10 December 2021).

40. Zhang, Y.; Kang, M.S.; Lamkey, K.R. DIALLEL-SAS05: A Comprehensive program for Griffing's and Gardner-Eberhart Analyses. Agron. J. 2005, 97, 1097-1106. [CrossRef]

41. Jinks, J.L.; Jones, R.M. Estimation of the components of heterosis. Genetics 1958, 43, 223-234. [CrossRef]

42. Griffing, B. Analysis of quantitative gene action by constant parent regression and related techniques. Genetics 1950, 35, 303-321. [CrossRef]

43. Singh, V.; Vashisht, V.K. Heterosis and combining ability for yield in muskmelon (Cucumis melo L.). Int. J. Curr. Microbiol. Appl. Sci. 2018, 7, 2996-3006. [CrossRef]

44. Feyzian, E.; Dehghani, H.; Rezai, A.M.; Javaran, M.J. Diallel cross analysis for maturity and yield-related traits in melon (Cucumis melo L.). Euphytica 2009, 168, 215-223. [CrossRef]

45. Hassan, W.H.A.; Gad, A.A.; El-salam, M.M.A.; Ismail, H.E.M. Gene action and heterosis of muskmelon. Zagazig J. Agric. Res. 2018, 45, 1953-1961. [CrossRef]

46. Neto, J.G.C.; Ferreira, K.T.C.; de Aragão, F.A.S.; Antônio, R.P.; de Sousa Nunes, G.H. Potential of parents and hybrids experimental of the yellow melon. Cienc. Rural 2020, 50, 1-9. [CrossRef]

47. Saha, K.; Mishra, S.; Choudhary, H.; Mahapatra, S. Estimates of genetic component of variation in muskmelon (Cucumis melo L.). Int. J. Curr. Microbiol. App. Sci. 2018, 7, 1144-1151. [CrossRef]

48. Badami, K.; Daryono, B.S.; Amzeri, A.; Khoiri, S. Combining ability and heterotic studies on hybrid melon (Cucumis melo L.) populations for fruit yield and quality traits. SABRAO J. Breed. Genet. 2020, 52, 402-417.

49. Mohammadi, R.; Dehghani, H.; Karimzadeh, G. Genetic analysis of yield components, early maturity and total soluble solids in cantaloupe (Cucumis melo L. subsp. melo var cantalupensis Naudin. Yüzüncü Yıl Üniversitesi Tartm Bilim. Derg. 2014, 24, 79-86. [CrossRef]

50. Rolania, S.; Fageria, M.S. Heterosis and combining ability evaluation for yield, quality, and fruit fly resistance in muskmelon. Int J. Curr. Microbiol. Appl. Sci. 2018, 7, 902-915. [CrossRef]

51. Pouyesh, A.; Lotfi, M.; Ramshini, H.; Karami, E.; Shamsitabar, A.; Armiyoun, E. Genetic analysis of yield and fruit traits in cantaloupe cultivars. Plant Breed. 2017, 136, 569-577. [CrossRef]

52. Kalb, T.J., II; Davis, D.W. Evaluation of combining ability, heterosis, and genetic variance for fruit quality characteristics in bush muskmelon. Am. Soc. Hortic. Sci. 1984, 109, 411-415.

53. Zalapa, J.E.; Staub, J.E.; McCreight, J.D. Variance component analysis of plant architectural traits and fruit yield in melon. Euphytica 2008, 162, 129-143. [CrossRef] 
54. Saftner, R.A.; Lester, G.E. Sensory and analytical characteristics of a novel hybrid muskmelon fruit intended for the fresh-cut industry. Postharvest Biol. Technol. 2009, 51, 327-333. [CrossRef]

55. Javanmard, T.; Saleh-Abadi, F.S.; Bihamta, M.R. Estimation of some genetic parameters through generation mean analysis in melon. Indian J. Anim. Res. 2018, 52, 619-624. [CrossRef]

56. Sharma, S.P.; Leskovar, D.I.; Crosby, K.M.; Ibrahim, A.M.H. GGE biplot analysis of genotype-by-environment interactions for melon fruit yield and quality traits. HortScience 2020, 55, 533-542. [CrossRef]

57. Sedera, A.; Badr, L.A.A.; Nagar, M.M.E.; Ayad, M.A.M. Inheritance of some fruit quality characteristics of melon. Middle East J. Agric. Res. 2016, 5, 789-809.

58. Perchepied, L.; Pitrat, M. Polygenic inheritance of partial resistance to Fusarium oxysporum f. sp. melonis race 1.2 in melon. Phytopathology 2004, 94, 1331-1336. [CrossRef] [PubMed]

59. Herman, R.; Perl-Treves, R. Characterization and inheritance of a new source of resistance to Fusarium oxysporum f. sp. melonis race 1.2 in Cucumis melo. Plant Dis. 2007, 91, 1180-1186. [CrossRef] [PubMed]

60. Chikh-Rouhou, H.; González-Torres, R.; Oumouloud, A.; Alvarez, J.M. Inheritance of race 1.2 Fusarium wilt resistance in four melon cultivars. Euphytica 2011, 182, 177-186. [CrossRef]

61. Noormohammadi, Z.; Hasheminejad-Ahangarani Farahani, Y.; Sheidai, M.; Ghasemzadeh-Baraki, S.; Alishah, O. Genetic diversity analysis in opal cotton hybrids based on SSR, ISSR, and RAPD markers. Genet. Mol. Res. 2013, 12, 256-269. [CrossRef] [PubMed] 